\title{
QUEEN'S
UNIVERSITY
BELFAST
}

\section{Prediction of limit cycle oscillations under uncertainty using a Harmonic Balance method}

Hayes, R., \& Marques, S. P. (2015). Prediction of limit cycle oscillations under uncertainty using a Harmonic Balance method. Computers \& Structures, 148, 1-13. https://doi.org/10.1016/j.compstruc.2014.10.010

\author{
Published in: \\ Computers \& Structures
}

\section{Document Version:}

Peer reviewed version

Queen's University Belfast - Research Portal:

Link to publication record in Queen's University Belfast Research Portal

\section{Publisher rights}

This is the author's version of a work that was accepted for publication in Computers \& Structures. Changes resulting from the publishing process, such as peer review, editing, corrections, structural formatting, and other quality control mechanisms may not be reflected in this document. Changes may have been made to this work since it was submitted for publication. A definitive version was subsequently published in Computers \& Structures, Volume 148, February 2015, doi:10.1016/j.compstruc.2014.10.010

\section{General rights}

Copyright for the publications made accessible via the Queen's University Belfast Research Portal is retained by the author(s) and / or other copyright owners and it is a condition of accessing these publications that users recognise and abide by the legal requirements associated with these rights.

Take down policy

The Research Portal is Queen's institutional repository that provides access to Queen's research output. Every effort has been made to ensure that content in the Research Portal does not infringe any person's rights, or applicable UK laws. If you discover content in the Research Portal that you believe breaches copyright or violates any law, please contact openaccess@qub.ac.uk. 


\section{Elsevier Editorial System(tm) for Computers and Structures Manuscript Draft}

Manuscript Number: CAS-D-13-00860R1

Title: Prediction of Limit Cycle Oscillations under Uncertainty using a Harmonic Balance Method

Article Type: Research Paper

Keywords: Aeroelasticity;

Harmonic Balance;

Limit-Cycle Oscillations;

Uncertainty;

Bifurcation;

Nonlinear

Corresponding Author: Dr. Simao Marques,

Corresponding Author's Institution: Queen's University Belfast

First Author: Richard Hayes, MEng

Order of Authors: Richard Hayes, MEng; Simao Marques

Abstract: The Harmonic Balance method is an attractive solution for computing periodic responses and can be an alternative to time domain methods, at a reduced computational cost. The current paper investigates using a Harmonic Balance method for simulating limit cycle oscillations under uncertainty. The Harmonic Balance method is used in conjunction with a nonintrusive polynomial-chaos approach to propagate variability and is validated against Monte Carlo analysis. Results show the potential of the approach for a range of nonlinear dynamical systems, including a full wing configuration exhibiting supercritical and subcritical bifurcations, at a fraction of the cost of performing time domain simulations. 


\title{
Prediction of Limit Cycle Oscillations under Uncertainty using a Harmonic Balance Method
}

\author{
Richard Hayes, Simão P. Marques ${ }^{1}$, \\ School of Mechanical and Aerospace Engineering \\ Queen's University Belfast, Belfast, UK, BT9 5AH
}

\begin{abstract}
The Harmonic Balance method is an attractive solution for computing periodic responses and can be an alternative to time domain methods, at a reduced computational cost. The current paper investigates using a Harmonic Balance method for simulating limit cycle oscillations under uncertainty. The Harmonic Balance method is used in conjunction with a nonintrusive polynomial-chaos approach to propagate variability and is validated against Monte Carlo analysis. Results show the potential of the approach for a range of nonlinear dynamical systems, including a full wing configuration exhibiting supercritical and subcritical bifurcations, at a fraction of the cost of performing time domain simulations.
\end{abstract}

Keywords: Aeroelasticity, Harmonic Balance, Limit Cycle Oscillations, Uncertainty, Bifurcation, Nonlinear.

\section{Introduction}

As design complexity increases, new materials and novel technologies are introduced to new airframes, empirical methods become increasingly difficult to apply, hence a clear need for physics based modelling tools has emerged. Aeroelasticity in particular is a good illustration of this trend and a need for physics based modelling tools has been identified by Noll et al[1]. Furthermore, predicting the aeroelastic stability of an aircraft should also identify the consequences of variability or uncertainty in model parameters, as discussed by Pettit[2]. Marques et al demonstrated the significant impact of structural variability on transonic flutter predictions[3, 4]. When nonlinearities are present, the amplitude of oscillations can become limited and limit 
cycle oscillations are observed. This is a problem of considerable practical interest and is well documented for in-service aircraft $[5,6]$. When nonlinearities are de-stabilizing (softening) a subcritical limit-cycle exists. As discussed by Stanford and Beran[7], unstable LCOs can occur below the flutter speed and lead to a hysteretic phenomenon. This type of instability is extremely undesirable because as the flutter speed is reached, the amplitude increases suddenly and significantly, as the the speed drops below the flutter point, the LCO will persist.

The presence of nonlinearities, either structural or aerodynamic, poses additional challenges both in terms of complexity and computational resources, these requirements can be exacerbated by the need to quantify the uncertainty due to unknown or variable parameters. Hence, several efforts have been made to address both these issues.

Reduced order modelling is a technique widely utilised to ease the computational burden associated with high-fidelity unsteady simulations, required to capture nonlinear effects. Proper orthogonal decomposition (POD) is commonly used to compress high order data $[8,9]$ and has been implemented in a reliability-based design optimisation framework (RBDO) for aeroelastic problems[10]. Volterra series can be used to model nonlinear responses with historic consideration, hence suitable for transient problems[11]. Recently, recurrent artificial neural networks (ANN), were applied to replicate an input-output relationship and can be used for nonlinear problems, such as LCOs[12], provided the model is sufficiently trained. System identification techniques using describing functions are another alternative to capture unsteady aerodynamic effects in dynamic aeroelastic problems [13, 14]. The common limitation of the methods mentioned above is the sacrifice of physical accuracy and parameter space associated with the reduction process, rendering the ROM unreliable outside the limits of the original data. The application of ROMs to uncertainty quantification (UQ) problems is in principle possible, however, the associated increase in the parameter space would require additional computational resources to generate suitable ROMs.

Two promising approaches which do not compromise the underlying physics of the oscillatory behaviour and have been applied to LCOs are: model reduction techniques based on the centre manifold theorem[15] and frequencybased techniques (finite-difference cyclic methods[16], spectral elements in time[17] and Harmonic Balance (HB) methods[6, 18, 19]). Although the HB method employs global basis functions resulting in system matrices with no sparsity, it offers better temporal convergence than spectral element and 
cyclic methods[20]. Additionally, convergence problems can occur for the spectral element method during the transition between unstable and stable branches of a subcritical LCO[7], the HB method does not encounter this problem. An overview of different variations of the Harmonic Balance method, such as high-dimensional, incremental, or elliptic HB methods is given by Dimitriadis[21].

The growth in complexity associated with classical HB method for higher order nonlinear terms render it inefficient for most practical problems[18]. The High-Dimensional Harmonic Balance (HDHB) method can simplify the treatment of nonlinearities thus making it scalable for more complex problems and can subsequently offer over one order of magnitude reduction in cost [22]. The benefits of the Harmonic Balance approach deteriorate as the number of harmonics retained to solve the problem increase[23].

As for flutter, LCOs are sensitive to parametric variability, which makes the use of stochastic tools attractive to this problem. Beran et al[24] applied several UQ techniques to an aerofoil LCO problem, where variability was propagated using time domain and cyclic methods. To overcome the difficulties with applying stochastic methods such as Probabilistic Collocation to long time integration problem, Witteveen et al[25], re-cast LCO time domain results as a function of the resultant frequency. More recently, Le Meitour et al [26] used a non-intrusive, adaptive formulation of a generalised Polynomial Chaos Expansion (PCE) approach to 2-dimensional LCO problems, the adaptive formulation allowed for the PCE method to give reliable answers in the presence of discontinuities such as supercritical bifurcations.

In this work, an HDHB formulation is exploited to determine the LCO conditions without incurring the costs of time-accurate simulations; the paper then investigates the practicality of using the HDHB approach to propagate parametric variability using a Non-Intrusive Polynomial Chaos (NIPC) approach. The paper will first summarize the HDHB formulation, this is followed by the description of the probabilistic approach based on non-intrusive PCE. The impact of variability on the responses amplitudes and motion frequency is assessed and compared against Monte Carlo (MC) results (using both time domain and HDHB methods).

\section{Harmonic Balance Formulation}

The HB formulation used in this work was proposed by Hall et al[22] for time-periodic flow problems, this methodology was adapted to nonlin- 
ear dynamical systems by Liu et al[27] and is summarised next. Consider a dynamic system with a nonlinearity in stiffness whose behaviour can be described using a simple equation of motion given by:

$$
\mathbf{M} \ddot{x}+\mathbf{C} \dot{x}+\mathbf{K} x+\mathbf{K}_{n l}(x)=\mathbf{F}(x, \dot{x}, \ddot{x}, t)
$$

Matrices $\mathbf{M}, \mathbf{C}$ and $\mathbf{K}$ describe the mass, damping and linear stiffness properties of the system respectively and $\mathbf{K}_{n l}(x)$ is the nonlinear component of the stiffness restoring force. The external force, $\mathbf{F}$ can be a function of the motion of the system and/or time. Here consider the external force to be, $\mathbf{F}=\sin (\omega t)$. The solution of eq. (1) can be approximated to be a truncated Fourier series of $N_{H}$ harmonics with a fundamental frequency $\omega$.

$$
x(t) \approx \hat{x}_{0}+\sum_{n=1}^{N_{H}}\left(\hat{x}_{2 n-1} \cos (n \omega t)+\hat{x}_{2 n} \sin (n \omega t)\right)
$$

The first and second derivatives of $x(t)$ with respect to time can be found to be:

$$
\begin{gathered}
\dot{x}(t) \approx \sum_{n=1}^{N_{H}}\left(-n \omega \hat{x}_{2 n-1} \sin (n \omega t)+n \omega \hat{x}_{2 n} \cos (n \omega t)\right) \\
\ddot{x}(t) \approx \sum_{n=1}^{N_{H}}\left(-(n \omega)^{2} \hat{x}_{2 n-1} \cos (n \omega t)-(n \omega)^{2} \hat{x}_{2 n} \sin (n \omega t)\right)
\end{gathered}
$$

By substituting the Fourier series back into eq. (1) and collecting terms associated with each harmonic, a system of equations can be assembled that relate the system's dynamic properties with the Fourier coefficients. This algebraic system consists of $2 N_{H}+1$ equations which can be conveniently displayed in vector form:

$$
\left(\mathbf{M} \omega^{2} \mathbf{A}^{2}+\mathbf{C} \omega \mathbf{A}+\mathbf{K I}\right) \hat{\mathbf{Q}}+\hat{\mathbf{Q}}_{n l}-\mathbf{F} \hat{\mathbf{H}}=0
$$

where:

$$
\hat{\mathbf{Q}}=\left\{\begin{array}{c}
\hat{x}_{0} \\
\hat{x}_{1} \\
\hat{x}_{2} \\
\hat{x}_{3} \\
\vdots \\
\hat{x}_{2 N_{H}}
\end{array}\right\}_{2 N_{H}+1}, \hat{\mathbf{Q}}_{n l}=\left\{\begin{array}{c}
\hat{K}_{n l 0} \\
\hat{K}_{n l 1} \\
\hat{K}_{n l 2} \\
\hat{K}_{n l 3} \\
\vdots \\
\hat{K}_{n l 2 N_{H}}
\end{array}\right\}_{2 N_{H}+1} \quad \text { and } \hat{\mathbf{H}}=\left\{\begin{array}{c}
0 \\
0 \\
1 \\
0 \\
\vdots \\
0
\end{array}\right\}_{2 N_{H}+1}
$$


Matrix A reconstructs the Fourier series from the harmonic balancing and is shown in Appendix A. The nonlinear term Fourier coefficients, $\hat{\mathbf{K}}_{n l}$ are computed using discrete Fourier transforms (DFT). Computing these terms analytically can be cumbersome for certain types of nonlinearities such as high order polynomial terms or fluxes in CFD problems[22]. The HDHB method overcomes these issues by casting the problem in the time domain where the Fourier coefficients are related to $2 N_{H}+1$ equally spaced subtime levels throughout one temporal period using a constant transformation matrix which yields:

$$
\hat{\mathbf{Q}}=\mathbf{E} \tilde{\mathbf{Q}}, \hat{\mathbf{Q}}_{n l}=\mathbf{E} \tilde{\mathbf{Q}}_{n l} \text { and } \hat{\mathbf{H}}=\mathbf{E} \tilde{\mathbf{H}}
$$

where:

$$
\tilde{\mathbf{Q}}=\left\{\begin{array}{c}
x\left(t_{0}\right) \\
x\left(t_{1}\right) \\
\vdots \\
x\left(t_{2 N_{H}}\right)
\end{array}\right\}, \tilde{\mathbf{Q}}_{n l}=\left\{\begin{array}{c}
K_{n l}\left(x\left(t_{0}\right)\right) \\
K_{n l}\left(x\left(t_{1}\right)\right) \\
\vdots \\
K_{n l}\left(x\left(t_{2 N_{H}}\right)\right)
\end{array}\right\} \text { and } \tilde{\mathbf{H}}=\left\{\begin{array}{c}
\sin t_{0} \\
\sin t_{1} \\
\vdots \\
\sin t_{2 N_{H}}
\end{array}\right\}
$$

and

$$
t_{i}=\frac{i 2 \pi}{2 N_{H}+1}\left(i=0,1, \ldots, 2 N_{H}\right)
$$

Expressions for the transformation matrix $\mathbf{E}$ and its inverse $\mathbf{E}^{-1}$ which can be used to relate the time domain variables back to the Fourier coefficients ie. $\tilde{\mathbf{Q}}=\mathbf{E}^{-1} \hat{\mathbf{Q}}$ are shown in Appendix A. Using these transformation matrices, the system in eq. (5) can be cast in the time domain as:

$$
\left(\mathbf{M} \omega^{2} \mathbf{D}^{2}+\mathbf{C} \omega \mathbf{D}+\mathbf{K I}\right) \tilde{\mathbf{Q}}+\tilde{\mathbf{Q}}_{n l}-\mathbf{F} \tilde{\mathbf{H}}=0=\mathbf{R}
$$

where $\mathbf{D}=\mathbf{E}^{-1} \mathbf{A E}$. Equation (8) represents the HDHB system and can be solved using either pseudo-time marching or Newton-Raphson approaches. Here, the latter is employed:

$$
\mathbf{S}^{n+1}=\mathbf{S}^{n}-\lambda \mathbf{J}^{-1} \mathbf{R}^{n}
$$

where $\mathbf{S}^{n}$ is the solution vector at iteration $n, \lambda$ is a relaxation parameter for increased stability. The inverse Jacobian of the system, $\mathbf{J}^{-1}$, is numerically approximated using finite-differences[28] and $\mathbf{R}^{n}$ is the residual of eq. (8) at 
iteration $n$, ie.

$$
\mathbf{S}^{n}=\left\{\begin{array}{c}
\hat{x}_{0} \\
\hat{x}_{1} \\
\vdots \\
\hat{x}_{2 N_{H}}
\end{array}\right\}^{n}, \mathbf{R}^{n}=\left\{\begin{array}{c}
R_{0} \\
R_{1} \\
\vdots \\
R_{2 N_{H}}
\end{array}\right\}^{n} \text { and } \mathbf{J}=\left[\begin{array}{ccc}
\frac{\partial R_{0}}{\partial \hat{x}_{0}} & \cdots & \frac{\partial R_{0}}{\partial \hat{x}_{2 N_{H}}} \\
\vdots & \ddots & \vdots \\
\frac{\partial R_{2 N_{H}}}{\partial \hat{x}_{0}} & \cdots & \frac{\partial R_{2 N_{H}}}{\partial \hat{x}_{2 N_{H}}}
\end{array}\right]
$$

\section{Stochastic Modelling}

The system of equations shown in eq. (8) can be used to describe nonlinear time periodic dynamical systems and quantities such as frequency and amplitude are easily extracted. This enables the HDHB method to be an efficient way of propagating uncertainties in time periodic, nonlinear systems. The approach used in this paper is based on a non-intrusive polynomial chaos method, where sampled points from the parameter space are used to reconstruct a "polynomial expansion". Consider a generic system of equations with random input parameters given by:

$$
\mathcal{L}(x, t, \theta ; u)=f(x, t, \theta)
$$

where $u=u(x, t, \theta)$ is the solution and $f(x, t, \theta)$ is the source term. $\mathcal{L}$ is a general differential operator that may contain spatial, temporal, linear or nonlinear terms. The $\theta$ denotes a random event which may describe a system parameter, an initial or boundary condition, etc. In general $\theta \in$ $\Theta$, the set containing all possible outcomes. Therefore, $\theta$ can represent $M$ independent, continuous, uncertain parameters, in the cases reported here $\Theta=[0,1]^{M}$, where $\theta$ has mean values of $1 / 2$ and is uniformly distributed over $\Theta$. The solution is now a random process which can be represented as a truncated series of orthogonal polynomials, referred to as Polynomial Chaos Expansions[29]:

$$
\begin{gathered}
u(x, t, \theta)=a_{0} \Gamma_{0}+\sum_{i_{1}=1}^{P} a_{i_{1}} \Gamma_{1}\left(\varphi_{i_{1}}(\theta)\right)+\sum_{i_{1}=1}^{P} \sum_{i_{2}=1}^{i_{1}} a_{i_{1} i_{2}} \Gamma_{2}\left(\varphi_{i_{1}}(\theta), \varphi_{i_{2}}(\theta)\right)+ \\
\sum_{i_{1}=1}^{P} \sum_{i_{2}=1}^{i_{1}} \sum_{i_{3}=1}^{i_{2}} a_{i_{1} i_{2} i_{3}} \Gamma_{3}\left(\varphi_{i_{1}}(\theta), \varphi_{i_{2}}(\theta), \varphi_{i_{3}}(\theta)\right)+\ldots
\end{gathered}
$$

where $a_{i}$ are polynomial coefficients, $\Gamma_{p}$ represents basis functions, which are in fact orthogonal polynomials of order $p$, based on the random variable $\varphi_{i_{p}}=\left\{\varphi_{i_{1}}(\theta), \varphi_{i_{2}}(\theta), \ldots, \varphi_{i_{p}}(\theta)\right\}$. Equation (11) is usually written as:

$$
u(x, t, \theta)=\sum_{j=0}^{n_{b}} a_{j} \Psi_{j}(\varphi(\theta))
$$


The order-based indices correspond to each term $j$ in the new summation. There are several possible ways to obtain solutions to eq. (12), namely Galerkin Projection, Probabilistic Collocation, Point Collocation among others. Le Maître and Knio[30] provide a detailed review of techniques available. In this work, a regression based Point Collocation method is used, which requires sampling $u(x, t, \theta)$ for specific values of $\theta$ and forming the following system of equations:

$$
\underbrace{\left[\begin{array}{cccc}
\Psi_{0}\left(\varphi_{0}\right) & \Psi_{1}\left(\varphi_{0}\right) & \cdots & \Psi_{P}\left(\varphi_{0}\right) \\
\Psi_{0}\left(\varphi_{1}\right) & \Psi_{1}\left(\varphi_{1}\right) & \cdots & \Psi_{P}\left(\varphi_{1}\right) \\
\vdots & \vdots & \ddots & \vdots \\
\Psi_{0}\left(\varphi_{P}\right) & \Psi_{1}\left(\varphi_{P}\right) & \cdots & \Psi_{P}\left(\varphi_{P}\right)
\end{array}\right]}_{\mathbf{\Psi}} \underbrace{\left[\begin{array}{c}
a_{0} \\
a_{1} \\
\vdots \\
a_{P}
\end{array}\right]}_{\mathbf{a}}=\underbrace{\left[\begin{array}{c}
u_{0}(\theta) \\
u_{1}(\theta) \\
\vdots \\
u_{P}(\theta)
\end{array}\right]}_{\mathbf{u}}
$$

The solution of eq. (13) is given by:

$$
\mathbf{a}=\left(\Psi^{\mathrm{T}} \boldsymbol{\Psi}\right)^{-1} \boldsymbol{\Psi}^{\mathrm{T}} \mathbf{u}
$$

For a specified order of the polynomial, $P$, a set of $P+1$ vectors $\varphi_{i}$ for $i=0,1,2, \ldots, P$ are used in the approximation. The response values, $\mathbf{u}$, which can describe quantities such as amplitude or frequency, are obtained from sampling the probabilistic space used to define $\theta$, Hosder et al[31] suggest Latin hypercube sampling, but any sampling or design of experiments technique can be used. Oversampling is usually required, resulting in an overdetermined system and a least-squares minimization problem.

The orthogonal polynomials used to define the basis functions, $\Psi$, depend on type of the random variables, for example in the case of normal distributions, Hermite polynomials are typically used, whereas for uniformly distributed inputs Legendre polynomials are chosen[32]. With the regression model built, Monte Carlo analysis can be used with the regression model to obtain the statistic quantities of interest, usually $10^{4}-10^{6}$ samples suffice to converge the mean and standard deviation to engineering accuracy.

\section{Results}

In this study, the effects of uncertainties in initial conditions and structural parameters of various models exhibiting periodic oscillations is investigated:a one degree of freedom (dof) Duffing oscillator, a two dof aerofoil and a wing configuration. 


\subsection{Duffing Oscillator}

The problem defined in Liu et al[27] based on the Duffing oscillator serves as an initial test case to validate the current HDHB implementation. It contains one nonlinear parameter, a cubic stiffness variation. Following eq. (1), the terms are:

$$
\mathbf{M}=m, \mathbf{C}=2 \zeta \sqrt{k m}, \mathbf{K}=k, \mathbf{K}_{n l}(x)=\beta x^{3} \text { and } \mathbf{F}=F \sin (\omega t)
$$

where $\beta$ and $\zeta$ are the cubic stiffness and damping coefficients respectively. This system is is solved using an ordinary differential equation solver in MatLab (ODE45) and provides the reference time domain results used to assess the HDHB method. The application of the HDHB method yields the following system:

$$
\left(m \omega^{2} \mathbf{D}^{2}+2 \zeta \sqrt{k m} \omega \mathbf{D}+k \mathbf{I}\right) \tilde{\mathbf{Q}}_{x}+\beta \tilde{\mathbf{Q}}_{x}^{3}-F \tilde{\mathbf{H}}=0
$$

where $\tilde{\mathbf{Q}}_{x}$ and $\tilde{\mathbf{H}}$ are the solutions of displacement and the external force respectively at each time level. As before, $\mathbf{D}=\mathbf{E}^{-1} \mathbf{A E}$ with matrices $\mathbf{A}$ and $\mathbf{E}$ shown in Appendix A. Equation (16) is solved using the Newton-Raphson scheme shown in section 2 .

The impact of the number of harmonics employed by the HDHB method is demonstrated by the deterministic simulation of a Duffing oscillator as shown in Figure 1. It is clear that the HDHB solution will converge to the time domain result as the number of harmonics is increased and seven harmonics are sufficient in this case.

In the stochastic analysis of the Duffing oscillator two uncertain parameters are of interest, the magnitude and frequency of the external force. The additional structural parameters used here are: mass, $m=1 \mathrm{~kg}$, linear and cubic stiffness coefficients, $k=\beta=1$ and damping ratio, $\zeta=0.1$. The non-dimensional force magnitude, $F$ and frequency, $\omega$, were assigned mean values of 1.25 and 0.6 respectively and given a variation of $\pm 10 \%$ using a uniform distribution.

Table 1 shows the convergence rate of the MC simulation, using the HDHB solver and the motion's the peak-to-peak amplitude as the output measure; the samples were obtained using Latin hypercube sampling (LHS), $10^{4}$ samples give results of acceptable accuracy for this work and will be employed throughout this work.

The described PCE model was compared with the MC analysis using both time domain and HDHB approaches. A 5th order polynomial expansion was 


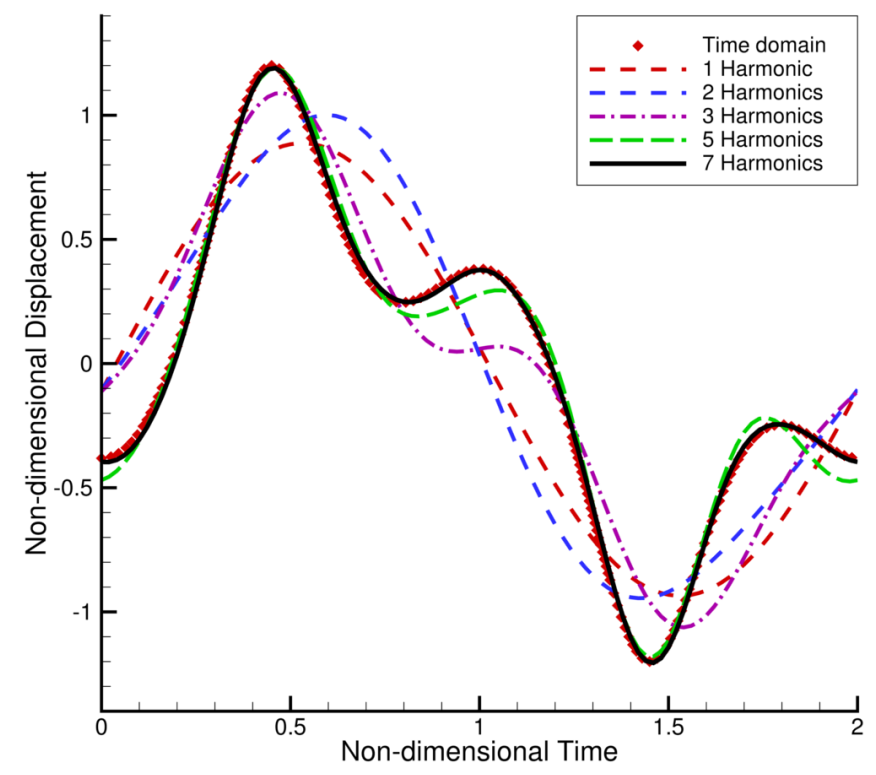

Figure 1: Number of harmonics convergence, $\omega=0.5$ and $F=1.25$

implemented requiring 44 samples. Figure 2 compares the response surface generated from the time accurate MC results with the HDHB-PCE. It can be seen that the amplitude of the response is affected by both the magnitude and frequency; it appears an increase in frequency, attenuates the impact of increases in the force magnitude. The histograms in Figure 2(c) show the frequencies of the amplitude and highlights the level of agreement between time domain MC and HDHB-PCE methods.

Table 2 shows the first two statistical moments for the motion's amplitude

Table 1: Amplitude mean and standard deviation for Duffing oscillator - Monte Carlo sample convergence

\begin{tabular}{c|c|c} 
No. of Samples & Mean & Std Deviation \\
\hline \hline 100 & 1.088160 & $4.835522 \times 10^{-2}$ \\
\hline 1,000 & 1.087853 & $4.631601 \times 10^{-2}$ \\
\hline 10,000 & 1.088065 & $4.701828 \times 10^{-2}$ \\
\hline 100,000 & 1.088070 & $4.702010 \times 10^{-2}$ \\
\hline \hline
\end{tabular}




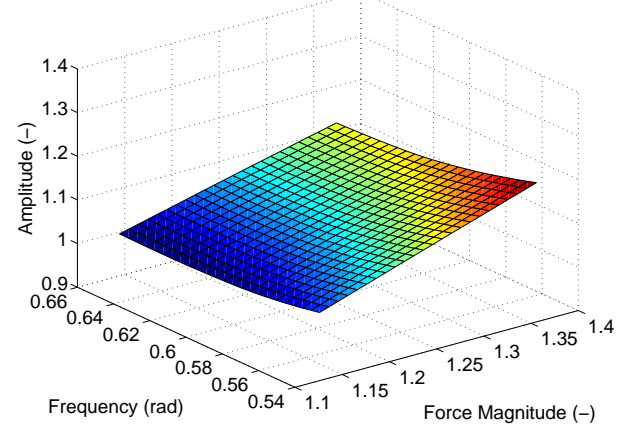

(a) MC Response Surface

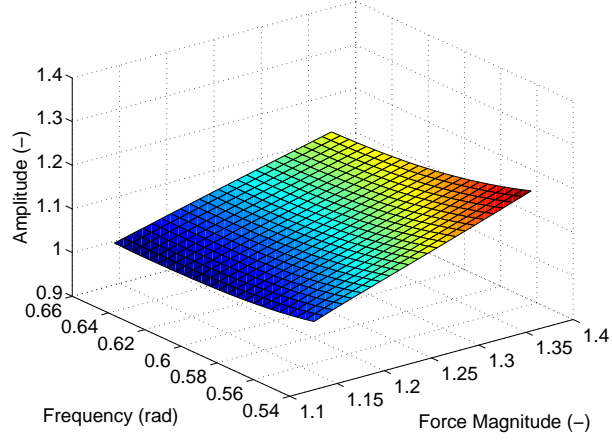

(b) HDHB PCE Response Surface
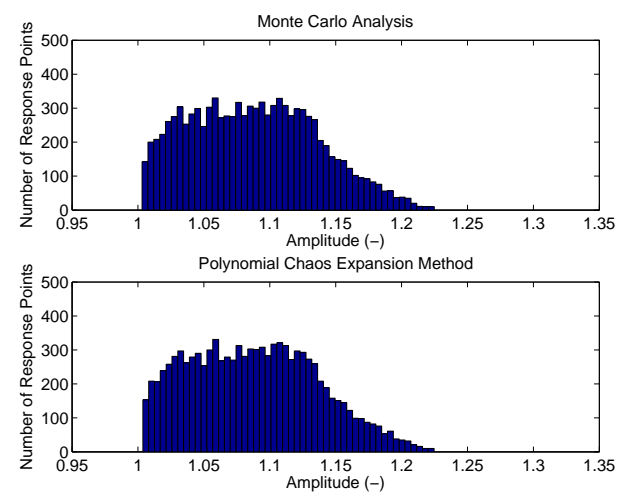

(c) Histograms of response solutions

Figure 2: Comparison of time domain MC analysis (MatLab ODE45) with HDHB PCE 
Table 2: Amplitude mean and standard deviation for Duffing oscillator

\begin{tabular}{l|c|c} 
& Time domain & HDHB method \\
\hline \hline MC mean & 1.088173 & 1.088065 \\
\hline PCE mean & 1.088032 & 1.088066 \\
\hline MC std deviation & $4.701390 \times 10^{-2}$ & $4.701828 \times 10^{-2}$ \\
\hline PCE std deviation & $4.703094 \times 10^{-2}$ & $4.707843 \times 10^{-2}$ \\
\hline \hline
\end{tabular}

obtained by the different methods, confirming the ability of the HDHB-PCE approach to capture the uncertainty in this system. Table 3 shows the computational cost of the problem.

\subsection{Pitch-Plunge Aerofoil}

In this section a typical aerofoil section restricted to pitching and plunging motions is analysed. The equations of motion for a pitch/plunge aerofoil with nonlinear restoring forces are shown in reference [33]. They can be displayed in the form of eq. (1), the terms are given as:

$$
\begin{gathered}
\mathbf{M}=\left[\begin{array}{cc}
x_{\alpha} & 1 \\
1 & \frac{x_{\alpha}}{r_{\alpha}^{2}}
\end{array}\right], \mathbf{C}=\left[\begin{array}{cc}
0 & \frac{2 \zeta_{\xi} \bar{\omega}}{V^{*}} \\
\frac{2 \zeta_{\alpha}}{V^{*}} \dot{\alpha} & 0
\end{array}\right], \mathbf{K}=\left[\begin{array}{cc}
0 & \frac{k_{\xi} \bar{\omega}^{2}}{V^{2}} \\
\frac{k_{\alpha}}{V^{* 2}} & 0
\end{array}\right], \\
x=\left\{\begin{array}{c}
\alpha \\
\xi
\end{array}\right\}, \mathbf{K}_{n l}=\left[\begin{array}{cc}
0 & \frac{\beta_{\xi} \bar{\omega}^{2}}{V^{* 2}} \\
\frac{\beta_{\alpha}}{V^{2}} & 0
\end{array}\right]\left\{\begin{array}{l}
\alpha^{3} \\
\xi^{3}
\end{array}\right\}+\left[\begin{array}{cc}
0 & \frac{\gamma_{\xi} \bar{\omega}^{2}}{V^{* 2}} \\
\frac{\gamma_{\alpha}}{V^{2}} & 0
\end{array}\right]\left\{\begin{array}{c}
\alpha^{5} \\
\xi^{5}
\end{array}\right\}, \\
\mathbf{F}=\left\{\begin{array}{l}
-\frac{C_{L}(\tau)}{\pi \mu}+\frac{b P(\tau)}{m V^{2}} \\
\frac{2 C_{M}(\tau)}{\pi \mu r_{\alpha}^{2}}+\frac{Q(\tau)}{m V^{2} r_{\alpha}^{2}}
\end{array}\right\}
\end{gathered}
$$

where $\xi$ is the non-dimensional plunge displacement of the elastic axis, $\alpha$ is the pitch, $C_{L}$ and $C_{M}$ correspond to the lift and pitching moment coefficients respectively, $P(\tau)$ and $Q(\tau)$ are external forces and moments respectively. After the introduction of four new variables, $w_{1}, w_{2}, w_{3}, w_{4}$ which partially 
describe the aerodynamic and external forces characterised by $\mathbf{F}$, the equations of motion can be written as:

$$
\begin{aligned}
& c_{0} \xi^{\prime \prime}+c_{1} \alpha^{\prime \prime}+c_{2} \xi^{\prime}+c_{3} \alpha^{\prime}+c_{4} \xi+c_{5_{\beta}} \xi^{3}+c_{5_{\gamma}} \xi^{5}+c_{6} \alpha+c_{7} w_{1}+c_{8} w_{2}+c_{9} w_{3}+c_{10} w_{4}=f\left(t^{*}\right) \\
& d_{0} \xi^{\prime \prime}+d_{1} \alpha^{\prime \prime}+d_{2} \alpha^{\prime}+d_{3} \alpha+d_{4_{\beta}} \alpha^{3}+d_{4_{\gamma}} \alpha^{5}+d_{5} \xi^{\prime}+d_{6} \xi+d_{7} w_{1}+d_{8} w_{2}+d_{9} w_{3}+d_{10} w_{4}=g\left(t^{*}\right)
\end{aligned}
$$

where $f\left(t^{*}\right)$ and $g\left(t^{*}\right)$ represent the remainder of the generalised aerodynamic forces and damp out with time hence are not part of the periodic solution. The time domain solutions are obtained by an explicit, fourth-order RungeKutta scheme[34, 35, 24, 36]. The nonlinearity is considered only in the pitch $\operatorname{dof}\left(c_{5_{\beta}}=c_{5_{\gamma}}=0\right)$. Expressions for the of the remainder coefficients can be found in [34]. Implementing the HDHB approach to eqs. (17) and (18), a system in the frequency domain can be created:

$$
\left\{\begin{array}{l}
\left(c_{0} \omega^{2} \mathbf{A}^{2}+c_{2} \omega \mathbf{A}+c_{4} \mathbf{I}\right) \hat{\mathbf{Q}}_{\xi}+\left(c_{1} \omega^{2} \mathbf{A}^{2}+c_{3} \omega \mathbf{A}+c_{6} \mathbf{I}\right) \hat{\mathbf{Q}}_{\alpha}+c_{7} \hat{\mathbf{Q}}_{w_{1}}+ \\
+c_{8} \hat{\mathbf{Q}}_{w_{2}}+c_{9} \hat{\mathbf{Q}}_{w_{3}}+c_{10} \hat{\mathbf{Q}}_{w_{4}}=0 \\
\left(d_{0} \omega^{2} \mathbf{A}^{2}+d_{5} \omega \mathbf{A}+d_{6} \mathbf{I}\right) \hat{\mathbf{Q}}_{\xi}+\left(d_{1} \omega^{2} \mathbf{A}^{2}+d_{2} \omega \mathbf{A}+d_{3} \mathbf{I}\right) \hat{\mathbf{Q}}_{\alpha}+d_{7} \hat{\mathbf{Q}}_{w_{1}}+ \\
+d_{8} \hat{\mathbf{Q}}_{w_{2}}+d_{9} \hat{\mathbf{Q}}_{w_{3}}+d_{10} \hat{\mathbf{Q}}_{w_{4}}+d_{4_{\beta}} \hat{\mathbf{Q}}_{\beta_{\alpha}}+d_{4_{\gamma}} \hat{\mathbf{Q}}_{\gamma_{\alpha}}=0 \\
\left(\omega \mathbf{A}+\epsilon_{1} \mathbf{I}\right) \hat{\mathbf{Q}}_{w_{1}}-\hat{\mathbf{Q}}_{\alpha}=0 \\
\left(\omega \mathbf{A}+\epsilon_{2} \mathbf{I}\right) \hat{\mathbf{Q}}_{w_{2}}-\hat{\mathbf{Q}}_{\alpha}=0 \\
\left(\omega \mathbf{A}+\epsilon_{1} \mathbf{I}\right) \hat{\mathbf{Q}}_{w_{3}}-\hat{\mathbf{Q}}_{\xi}=0 \\
\left(\omega \mathbf{A}+\epsilon_{2} \mathbf{I}\right) \hat{\mathbf{Q}}_{w_{4}}-\hat{\mathbf{Q}}_{\xi}=0
\end{array}\right.
$$

By substituting the last 4 equations of system 19 into the first 2 and replacing $\hat{\mathbf{Q}}_{\beta_{\alpha}}$ with $\mathbf{E}\left(\mathbf{E}^{-1} \hat{\mathbf{Q}}_{\alpha}\right)^{3}$ and $\hat{\mathbf{Q}}_{\gamma_{\alpha}}$ with $\mathbf{E}\left(\mathbf{E}^{-1} \hat{\mathbf{Q}}_{\alpha}\right)^{5}$ the system can be further reduced to[27]:

$$
\left(\mathbf{A}_{2}-\mathbf{B}_{2} \mathbf{B}_{1}^{-1} \mathbf{A}_{1}\right) \hat{\mathbf{Q}}_{\alpha}+d_{4_{\beta}} \mathbf{E}\left(\mathbf{E}^{-1} \hat{\mathbf{Q}}_{\alpha}\right)^{3}+d_{4_{\gamma}} \mathbf{E}\left(\mathbf{E}^{-1} \hat{\mathbf{Q}}_{\alpha}\right)^{5}=0
$$

where the matrices A. and B. are given by eqs. A.1-A.4.

\subsubsection{Supercritical Bifurcations}

Unlike the Duffing oscillator, the frequency of the response is not constrained, hence it must be treated as a variable in conjunction with the amplitude properties in order to capture the behaviour of the system. This is achieved by setting $\hat{\alpha}_{1}=0$ which will affect only the phase of the solution 
thus creating a system of $2 N_{H}+1$ equations with $2 N_{H}+1$ unknowns. The frequency can then be simultaneously solved along with the Fourier coefficients using the Newton-Raphson scheme. The solution and residual vectors are now given by:

$$
\mathbf{S}^{n}=\left\{\begin{array}{c}
\omega \\
\hat{\alpha}_{0} \\
\hat{\alpha}_{2} \\
\vdots \\
\hat{\alpha}_{2 N_{H}}
\end{array}\right\}^{n}, \mathbf{R}^{n}=\left\{\begin{array}{c}
R_{1} \\
R_{0} \\
R_{2} \\
\vdots \\
R_{2 N_{H}}
\end{array}\right\}^{n}
$$

The system parameters used for this case are $\bar{\omega}=0.2, \mu=100, a_{h}=$ $0.5, x_{\alpha}=0.25, r_{\alpha}=0.5, \zeta_{\alpha}=\zeta_{\xi}=0$ and $\gamma_{\alpha}=0 . \quad$ A $\pm 10 \%$ variation following a uniform distribution was imposed on the linear and cubic stiffness coefficients, with nominal values of 1.0 and 3.0 respectively. This nominal case was simulated over a range of velocities for different lengths of time, to determine convergence of the time domain solver as shown in Figure 3. As the velocity increases towards the the bifurcation point, the time domain solver requires increasingly longer time integration periods to damp out residual oscillations. For conditions beyond the bifurcation point, 2500 seconds suffice to achieve the final periodic orbit, hence this value was used for the nondeterministic calculations. Note that for the HDHB method, one harmonic is shown to be adequate for this investigation.

For a given velocity the system undergoes a supercritical Hopf-bifurcation, this is shown in Figure 4. The time domain and HDHB method show good agreement with exception to the bifurcation region due to the large cost associated with achieving a well-defined discontinuity. The presence of the bifurcation point requires a higher order polynomial capable of capturing the abrupt change in behaviour adequately. The effects of PCE order near the bifurcation point is shown by Figure 5, hence, an 11th order PCE requiring 194 samples was employed in the aerofoil investigation. The response frequency is shown in Figure 4. Note that there is a linear relationship between the linear stiffness coefficient and frequency for low values of linear stiffness, where LCOs exist. When no LCO exists, ie. amplitude is zero, frequencies with no physical meaning are generated which can have an adverse affect on the PCE performance. The histograms in Figure 6 reflect this limitation by the PCE when discontinuities are present.

Figure 7(a) shows the reference time domain response with respect to the 


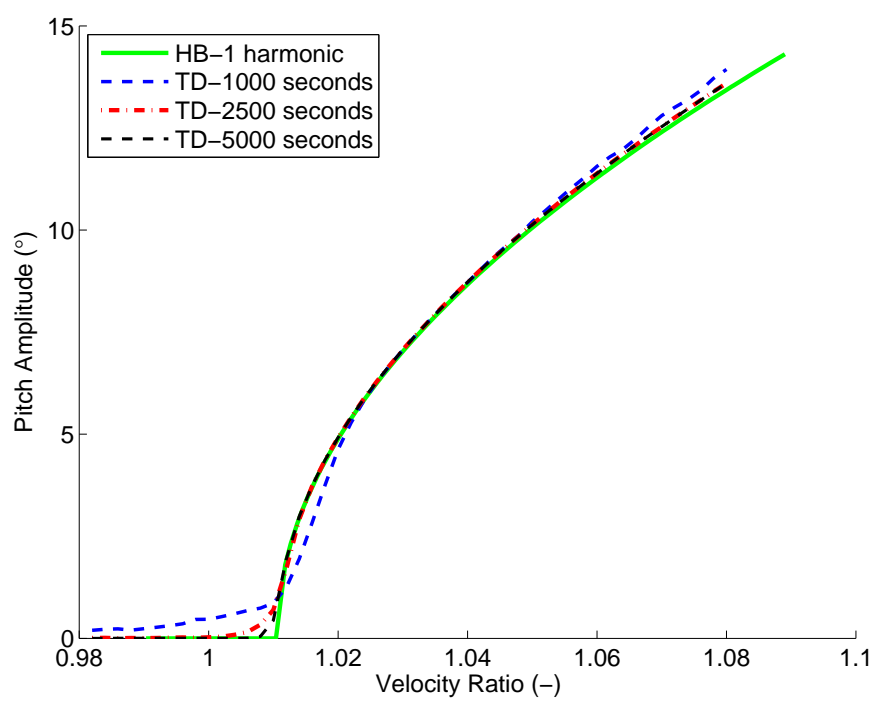

Figure 3: Convergence of time domain solver

deterministic case computed by a velocity sweep. It is evident that there is more variation present at lower velocities, this is due to increased sensitivity of the system close to the flutter speed (represented by the region of highest gradient). In this region, the linear stiffness has a larger effect on the stability of the system. The cubic stiffness impacts the amplitude as velocity increases because of the cubic relationship with displacement, but in this case the linear stiffness is the dominant factor. The HDHB method shows variations at all the velocities which are very similar to the time domain results. The PCE is shown to perform better at the higher velocities where only smooth behaviour is exhibited. The mean values extracted form the PCE analysis agree well with the MC time domain samples. The computational times for the aerofoil are shown in Table 3.

\subsubsection{Subcritical Bifurcations}

The subcritical case investigated here is similar to that used by Millman et al [37]. The desired subcritical behaviour is exhibited when the nonlinear term consists of a hardening pentic term, (+) and a softening cubic term, (-). The uncertain variables are the pentic stiffness coefficient, $\gamma_{\alpha}$, and the initial pitch amplitude, $\alpha_{0}$, with nominal values of 20 and $15^{\circ}$ respectively. $\mathrm{A} \pm 10 \%$ variation from a uniform distribution was assigned to the pentic stiffness coefficient, $\gamma_{\alpha}$, and a $\pm 50 \%$ variability of initial pitch amplitude, $\alpha_{0}$, was 
also added using a uniform distribution. The cubic stiffness coefficient was now softening, ie. $\beta_{\alpha}=-3$. PCE was not employed in the subcritical case as the hysteresis provides three disconnected response surfaces which cannot be sufficiently modelled without the partitioning of the random space[26]. Only the HDHB method was employed here as the time integration scheme that was implemented was unable to converge to the unstable branch of the subcritical LCO. Results are shown in Figure 8. The distribution of samples coincides with the different branches of the deterministic case, a large proportion of samples exhibit LCO behaviour below the bifurcation point. The variability in the chosen uncertain parameters has a more limited impact on the LCO amplitude than in the supercritical case, as shown by Figure 8. For a velocity ratio of 0.96 , Figure 9 (a) shows how lower values for the pentic stiffness coefficient lead to a wider range of initial pitch values

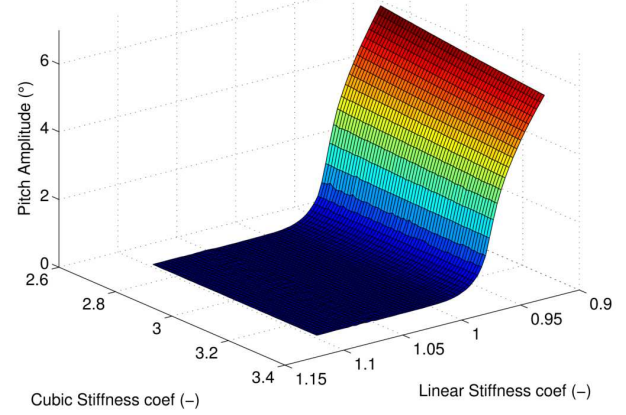

(a) Time domain $\mathrm{MC}$

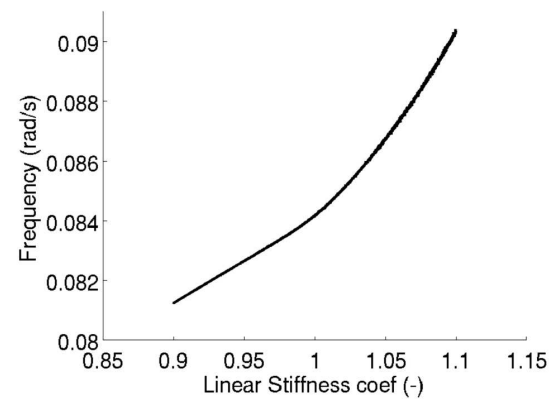

(c) TD MC scatter

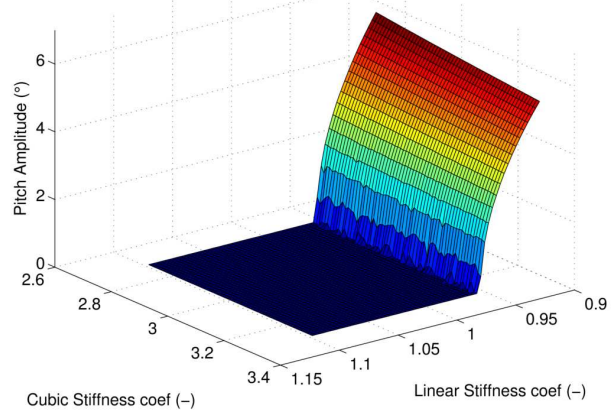

(b) HDHB method MC

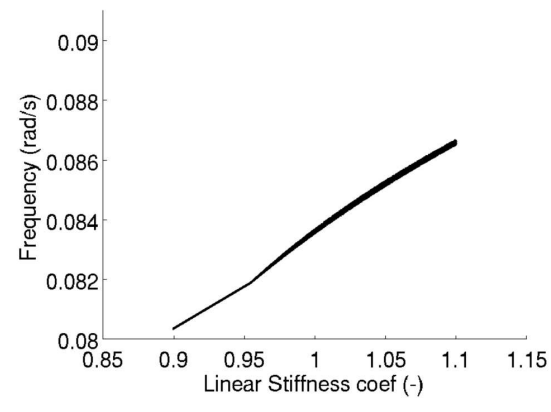

(d) HDHB MC scatter

Figure 4: Supercritical Bifurcation at Linear Flutter Speed 


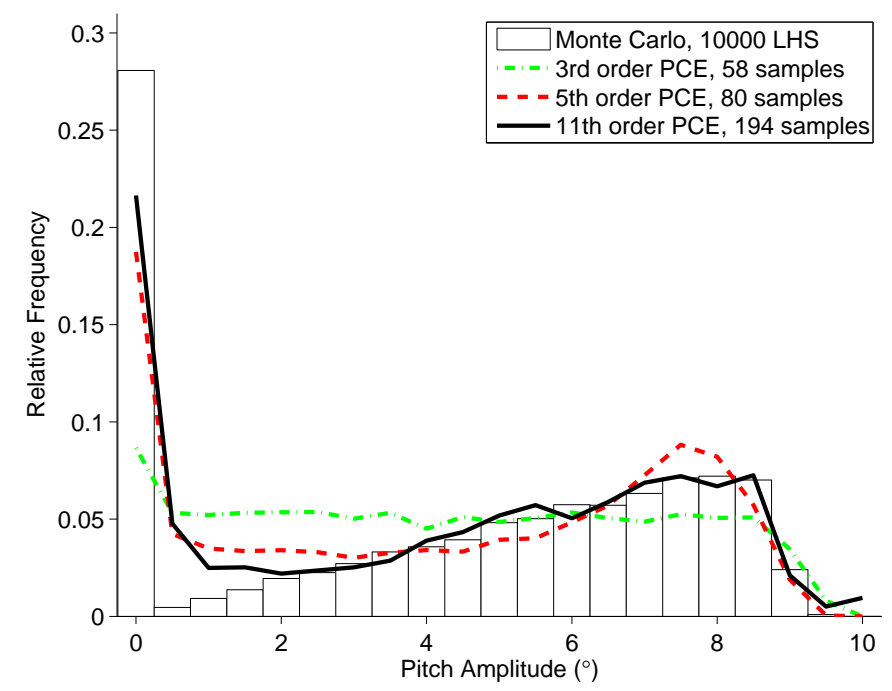

Figure 5: Histogram for Monte-Carlo and PCE, Supercritical Bifurcation at Velocity Ratio $=1.02$

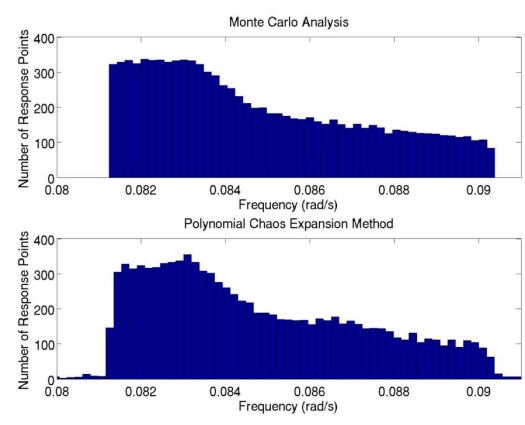

(a) Time domain

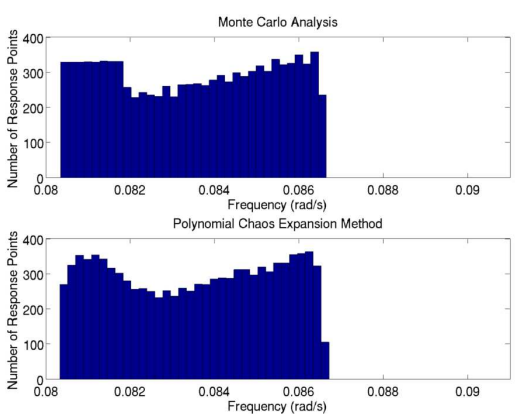

(b) HDHB method

Figure 6: Frequency Variation - Supercritical Bifurcation at Linear Flutter Speed 


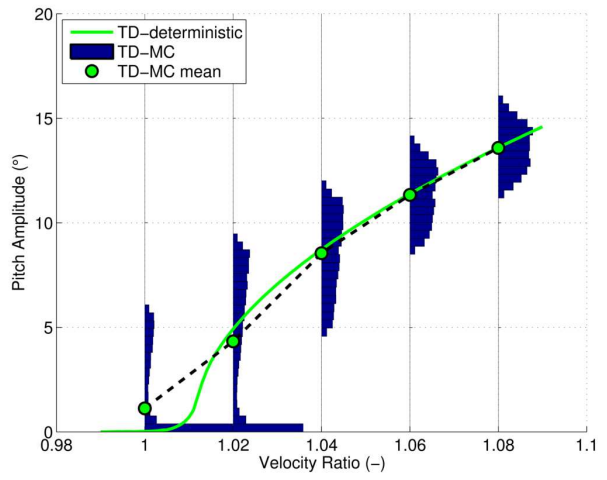

(a) Time domain MC

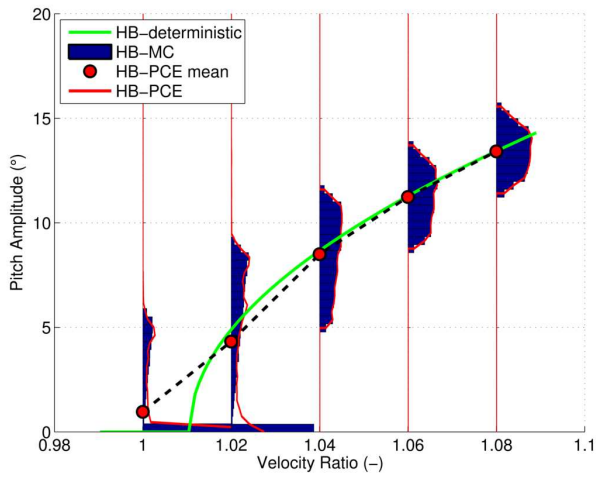

(b) HDHB method response

Figure 7: Supercritical Bifurcation Response

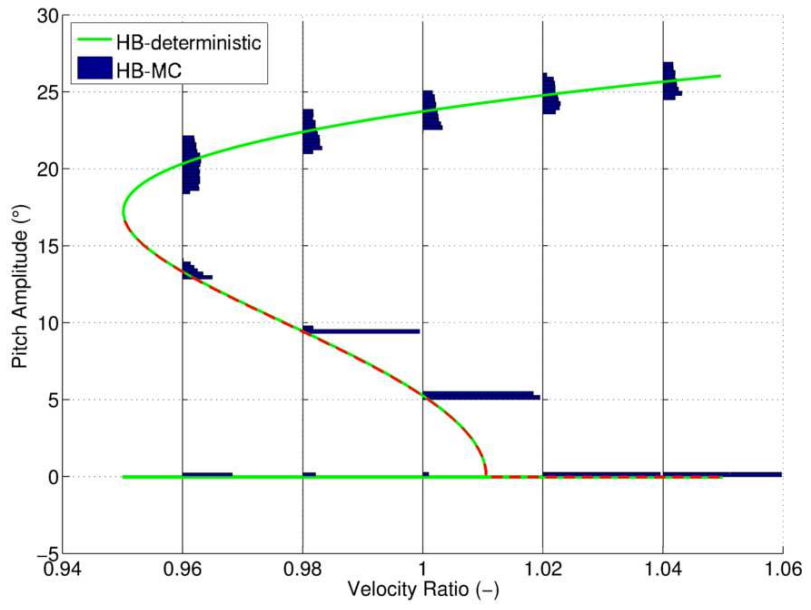

Figure 8: Subcritical Bifurcation, Monte-Carlo from HDHB 


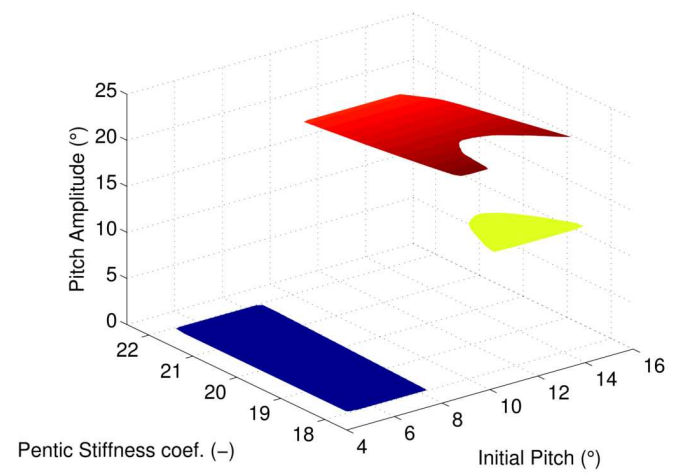

(a) LCO Amplitude

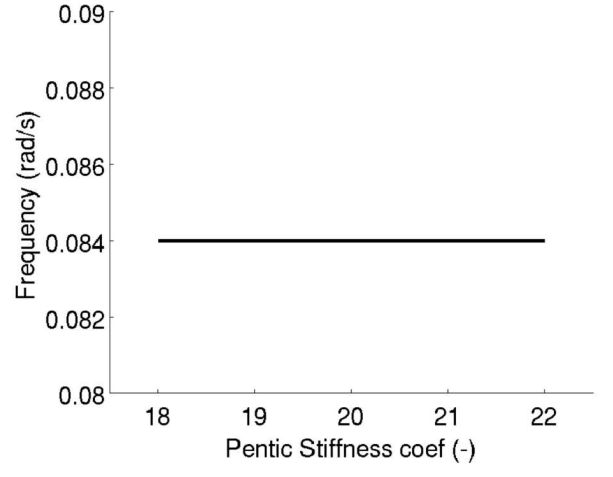

(b) LCO Frequency

Figure 9: Amplitude and Frequency Response, Velocity Ratio $=0.96$

to converge to the unstable branch, this subcritical behaviour is observed in the vicinity of the nominal initial pitch value. Figure $9(\mathrm{~b})$ shows the LCO frequency response of the aerofoil where the two LCO branches are characterised by the same frequency. Contrary to secondary bifurcations where two stable LCOs exist with different frequencies[19], the subcritical branch is unstable and is not a true physical solution, hence any point on this branch subsequently jumps to the stable upper branch.

\subsection{Goland wing}

The Goland wing provides a relatively simple 3D model exhibiting several complex aeroelastic phenomenon that are challenging to engineering prediction methods. The wing has a rectangular plan-form with a span of $20 \mathrm{ft}$ and $6 \mathrm{ft}$ chord. The finite element model of the heavy version of the Goland wing is described in [38] and is shown in Figure 10(a). The structural model used in this work includes localised nonlinearities between the tip store attachment stiffness and the wing. The nonlinearities are in the form of polynomial laws for spring elements in the translational $z$-direction $\left(\mathrm{K}_{\mathrm{z}}\right)$, and the rotational $y$-direction $\left(\mathrm{K}_{\mathrm{ry}}\right)$ degrees of freedom which were shown to be the most sensitive by reference[15]. The equations of motion for the Goland wing take the same form as eq. (1). The external forces, $\mathbf{F}$ acting on the wing are aerodynamic in nature. NASTRAN computes these forces in the modal domain using Doublet-Lattice method. Thus the wing is analysed in the modal domain, this also significantly reduces the complexity of the problem[39]. 


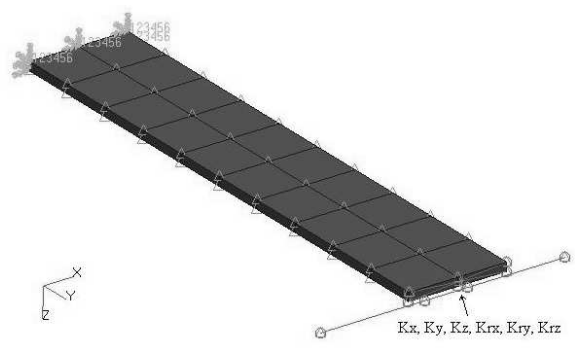

(a) Structural model

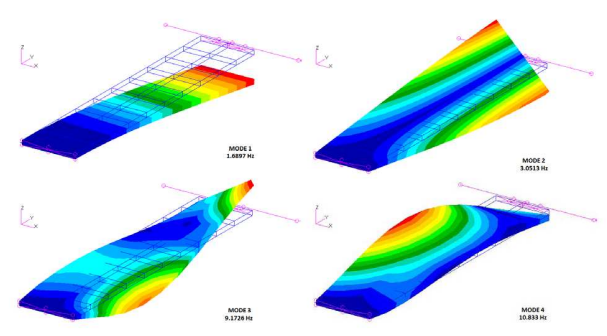

(b) First 4 mode shapes

Figure 10: Goland wing

The transformation between the physical and modal space[40] is given as $\{x\}=[\boldsymbol{\Phi}]\{\mathbf{q}\}$, where $\boldsymbol{\Phi}$ is the truncated matrix of eigenvectors, $\left(n_{m}\right.$ modes, extracted from NASTRAN) and $\mathbf{q}$ is a vector of $n_{m}$ modal coordinates.

The aerodynamic data is only computed for a range of discrete reduced frequencies so it is represented as a rational function to maintain validity for all values within this range[41]. For this investigation only four structural modes are considered and are shown in Figure 10(b). The system in the modal domain with modal coordinates, $\mathbf{q}$ is given as[15, 19]:

$$
\tilde{\mathbf{M}}_{\phi} \ddot{\mathbf{q}}+\tilde{\mathbf{C}}_{\phi} \dot{\mathbf{q}}+\tilde{\mathbf{K}}_{\phi} \mathbf{q}+\mathbf{K}_{n l}(\mathbf{q})=\frac{\rho V^{2}}{2} \sum_{i=1}^{n_{l}}\left[\mathbf{A}_{i+2}\right] \dot{\mathbf{q}}_{a_{i}}
$$

where $\tilde{\mathbf{M}}_{\phi}, \tilde{\mathbf{C}}_{\phi}$ and $\tilde{\mathbf{K}}_{\phi}$ are the aeroelastic system mass, damping and linear stiffness matrices, given by eq. B.1-B.3. $\mathbf{K}_{n l}(\mathbf{q})$ is the nonlinear force given by eq. B.4, A. represents the rationally approximated components of the generalised aerodynamic matrix extracted from NASTRAN. $\dot{\mathbf{q}}_{a_{i}}$ are augmented terms arising from the Laplace domain treatment of the generalised aerodynamic matrix and have the relationship:

$$
\ddot{\mathbf{q}}_{a_{i}}=\dot{\mathbf{q}}-\frac{V}{b} \eta_{i} \dot{\mathbf{q}}_{a_{i}}
$$

The state space equation which is solved in the time domain is constructed by combining eqs. (21) and (22), leading to:

$$
\dot{\mathbf{w}}+\mathbf{A}_{\mathbf{s}} \mathbf{w}+\mathbf{u}=0=\mathbf{R}
$$


where

$$
\mathbf{w}=\left\{\begin{array}{c}
\mathbf{q} \\
\dot{\mathbf{q}} \\
-\mathbf{A}_{3} \dot{\mathbf{q}}_{a_{1}} \\
-\mathbf{A}_{4} \dot{\mathbf{q}}_{a_{2}} \\
\vdots \\
-\mathbf{A}_{n_{l}+2} \dot{\mathbf{q}}_{a_{n_{l}}}
\end{array}\right\}, \quad \mathbf{u}=\left\{\begin{array}{c}
\mathbf{0} \\
\tilde{\mathbf{M}}_{\phi}^{-1} \boldsymbol{\Phi}^{\mathrm{T}} \mathbf{K}_{n l}(\mathbf{q}) \\
\mathbf{0} \\
\mathbf{0} \\
\vdots \\
\mathbf{0}
\end{array}\right\}
$$

and

$$
\mathbf{A}_{s}=\left[\begin{array}{cccccc}
\mathbf{0} & -\mathbf{I} & \mathbf{0} & \ldots & \ldots & \mathbf{0} \\
\tilde{\mathbf{M}}_{\phi}^{-1} \tilde{\mathbf{K}}_{\phi} & \tilde{\mathbf{M}}_{\phi}^{-1} \tilde{\mathbf{C}}_{\phi} & \tilde{\mathbf{M}}_{\phi}^{-1} \frac{\rho V^{2}}{2} \mathbf{I} & \ldots & \ldots & \tilde{\mathbf{M}}_{\phi}^{-1} \frac{\rho V^{2}}{2} \mathbf{I} \\
\mathbf{0} & \mathbf{A}_{3} & \frac{V}{b} \eta_{1} \mathbf{I} & \mathbf{0} & \ldots & \mathbf{0} \\
\mathbf{0} & \mathbf{A}_{4} & \mathbf{0} & \frac{V}{b} \eta_{2} \mathbf{I} & \ddots & \vdots \\
\vdots & \vdots & \vdots & \ddots & \ddots & \mathbf{0} \\
\mathbf{0} & \mathbf{A}_{n_{l}+2} & \mathbf{0} & \ldots & \mathbf{0} & \frac{V}{b} \eta_{n_{l}} \mathbf{I}
\end{array}\right]
$$

Applying the HDHB method to eq. (21) yields the system:

$$
\left[\tilde{\mathbf{M}}_{\phi} \mathbf{E}_{a c c}^{-1}+\tilde{\mathbf{C}}_{\phi} \mathbf{E}_{v e l}^{-1}+\tilde{\mathbf{K}}_{\phi} \mathbf{E}_{d e f}^{-1}\right] \hat{\mathbf{Q}}_{\phi}+\mathbf{E}_{d e f}^{-1} \hat{\mathbf{K}}_{n l}-\frac{\rho V^{2}}{2} \sum_{i=1}^{n_{l}}\left[\mathbf{A}_{i+2}\right] \mathbf{E}_{d e f}^{-1} \hat{\mathbf{Q}}_{a_{i}}=0
$$

$\mathbf{E}_{a c c}^{-1}, \mathbf{E}_{\text {vel }}^{-1}$ and $\mathbf{E}_{d e f}^{-1}$ are transformation matrices shown in Appendix B. The complexity of both the nonlinear stiffness force, $\left\{\hat{\mathbf{K}}_{n l}\right\}$ and the decomposed generalised aerodynamic vectors, $\left\{\hat{\mathbf{Q}}_{a_{i}}\right\}$ prevent the straightforward representation of these terms in the frequency domain. Subsequently system (24) is a time domain representation of the problem, where the nonlinear terms are represented as reconstructed Fourier series, the Fourier coefficients are formed using discrete Fourier transforms (DFT), computed numerically from the expressions shown in eqs. B.9-B.14. Equation (24) is solved simultaneously for $2 N_{H}+1$ equally spaced time steps across one period with $t_{i}=\frac{i 2 \pi}{2 n_{H}+1}, \quad\left(i=0,1, \ldots, 2 N_{H}\right)$ to maintain temporal accuracy. A NewtonRaphson scheme is employed and as in the previous cases; the response frequency is maintained as a variable by locking the cosine Fourier coefficient of the first harmonic of the first mode to zero.

\subsubsection{Supercritical Bifurcations}

Although global properties of the structure remain within a reasonably small tolerance, localised structural properties between different units can 
vary substantially[42]. The cubic stiffness nonlinearity is varied to represent this variability and is consistent with reference[15] where values over a range of orders of magnitude are chosen.

For this case the linear stiffness matrix, $\tilde{\mathbf{K}}_{\phi}$ is multiplied by a scalar linear stiffness coefficient given a value of $1 \pm 10 \%$, the cubic stiffness coefficient in the rotational dof about the $y$ axis was given a value of $10^{10}$. The cubic

stiffness in the $z$ direction, $\beta_{z}$ is defined by the expression, $10^{a_{z}^{\beta}}$, where the exponent, $a_{z}^{\beta}$ was the second uncertain parameter with a uniform distribution resulting in a variation of $10.95 \pm 5 \%$. The damping matrix, $\tilde{\mathbf{C}}_{\phi}$ is given a damping coefficient of 0.1 . The response surfaces defined by these uncertainties are displayed in Figure 11. As in the aerofoil case, the time domain method does not reach a fully periodic state within the allowed computational time, shown by the scatter of points in the response surface. Despite this, there is good agreement between the time domain and the HDHB method. It should be mentioned that, similar to the aerofoil case, the cubic stiffness nonlinearity has no impact on the frequency behaviour of the LCO.

Figure 12 shows the response of the Goland wing with respect to the corresponding deterministic case. One harmonic was used in the HDHB method for speed although fractionally smaller amplitudes are observed. In this case a 5th order PCE was used, requiring 74 samples. Figure 12(b) shows the HDHB-PCE agreeing with the MC results based on the time-integration formulation. As before the mean values of the response are accurately predicted. The simulations are ran for 100 seconds which is sufficient capture the important, high amplitude points. Results near the bifurcation point are slightly degraded, where the number of stable points is not well captured. The range of cubic stiffness values has resulted in a distribution of amplitudes which is skewed due to the concentration of stable points. It is also noted that the amplitude mode value remains almost constant, not necessarily following the increase in mean and deterministic results. Computational times for the Goland wing are shown in Table 3.

\subsubsection{Subcritical Bifurcations}

The subcritical behaviour is obtained by including a pentic stiffness term in the nonlinear restoring force vector as shown in eq. B.5. Note the cubic terms are now also softening terms as in the aerofoil case. In this case the pentic stiffness is given as: $\gamma_{z}=10^{a_{z}^{\gamma}}$ with a variation of $a_{z}^{\gamma}=16.5 \pm 5 \%$. The initial tip deflection, $x_{0}$ is also varied, ie. $x_{0}=0.75 \mathrm{in} \pm 100 \%$. The other 


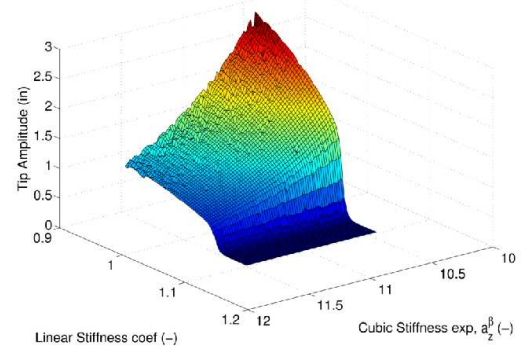

(a) Time domain MC

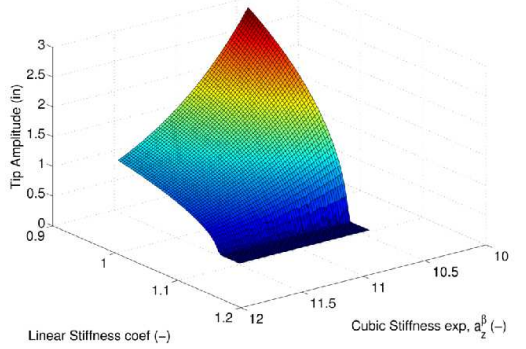

(b) HDHB method MC

Figure 11: Goland wing response at $690 \mathrm{fts}^{-1}$

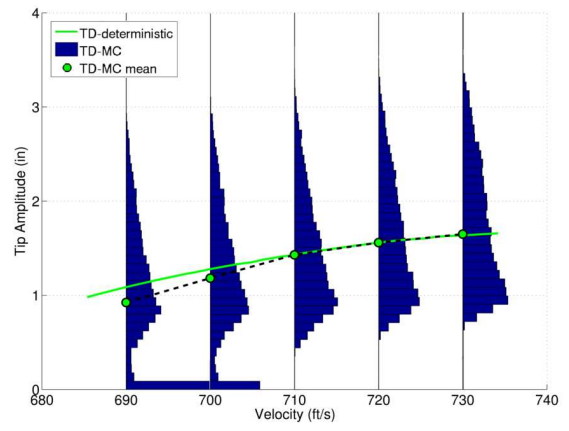

(a) Time domain $\mathrm{MC}$

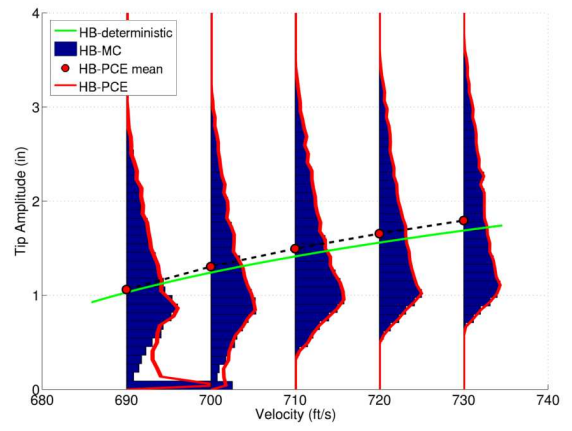

(b) HDHB

Figure 12: Goland Wing Supercritical LCO 


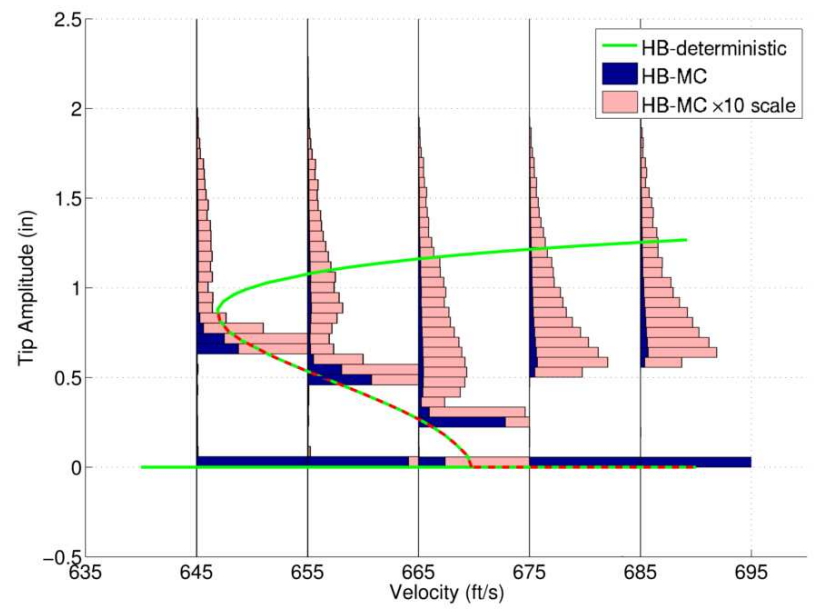

Figure 13: Goland Wing Subcritical LCO Amplitude, HDHB-MC

nonlinear stiffness coefficients are defined as: $\beta_{z}=10^{11.5}, \beta_{r y}=10^{10}$ and $\gamma_{r y}=10^{16.5}$. As in previous cases the variability is obtained from uniform distributions.

The MC response of the subcritical Goland wing is shown in Figure 13. The dashed line represents the unstable branches of the response. The light colour bars show the response magnified by a factor of 10, to help clarify the upper branch. Note that finite amplitude LCOs can still be encountered at lower velocities than possible in the deterministic case, furthermore it is at this velocity that the maximum amplitude is found, highlighting the impact of variability on the LCO subcritical behaviour. In some ways the subcritical behaviour of the wing is analogous to the aerofoil: as the pentic stiffness reduces, samples converge to the unstable branch, as shown by Figure 14 . The samples that converge to the stable branch are localised within a small vicinity of the nominal value of the pentic stiffness. These samples are responsible for the largest variations in amplitude, emphasising the sensitivity of the LCO to this parameter.

\section{Conclusions}

The influence of uncertainties in three nonlinear dynamical systems was investigated, a one dof Duffing oscillator, a two dof aerofoil and a multiple 


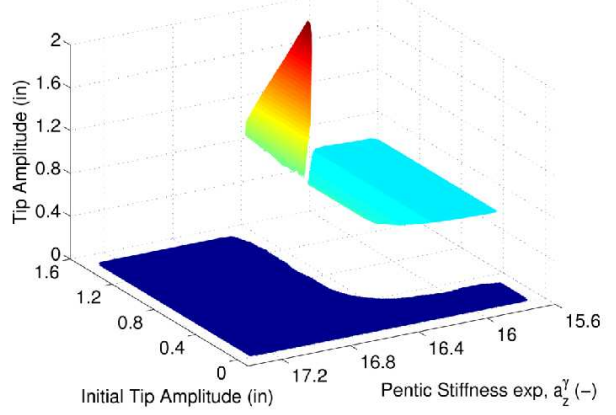

Figure 14: Goland Wing Subcritical LCO response at $645 \mathrm{fts}^{-1}$

dof 3D wing. Each system was simulated using time domain and HighDimensional Harmonic Balance methods where a non-intrusive polynomial chaos method was implemented and compared with Monte Carlo results. The HDHB method showed strong potential by modelling the system at a small fraction of the cost associated with time domain methods. The PCE method proved extremely effective for smooth response surfaces and showed significant reductions in cost in comparison with the Monte Carlo simulation. The limitations of PCE were highlighted when discontinuous behaviour was encountered as reproducing the bifurcation feature proved difficult, stability issues were encountered at the variable space boundaries. The subcritical behaviour of the aerofoil and Goland wing was investigated using the HDHB method which could reproduce the hysteretic unstable LCO branch. The unstable branch was characterised by lower values of the pentic stiffness and the same frequency as the stable upper branch as it is not a true physical solution. The variability of pentic stiffness can create the undesirable behaviour whereby large amplitude oscillations are found at velocities below

Table 3: Computational times

\begin{tabular}{l|c|c|c|c|c|c} 
& \multicolumn{6}{|c}{ Wall Clock (s) } \\
\hline \hline Case & \multicolumn{2}{|c|}{ Duffing } & \multicolumn{2}{c|}{ Aerofoil } & \multicolumn{2}{c}{ Goland wing } \\
\hline Method & MC & PCE & MC & PCE & MC & PCE \\
\hline Time domain & 9984 & 272 & 8042 & 142 & 83410 & 621 \\
\hline HDHB, NR & 123 & 5 & 136 & 9 & 27511 & 206 \\
\hline \hline
\end{tabular}


the bifurcation point.

\section{Acknowledgments}

The first author would like to thank the Department for Employment and Learning for Northern Ireland for funding his research. 


\section{Appendix A. Aerofoil Formulation}

The A matrix used in eqs. (5) and (19) is given as:

$$
\mathbf{A}=\left[\begin{array}{lllll}
0 & & & & \\
& \mathbf{N}_{1} & & & \\
& & \mathbf{N}_{2} & & \\
& & & \ddots & \\
& & & & \mathbf{N}_{N_{H}}
\end{array}\right]_{\left(2 N_{H}+1\right) \times\left(2 N_{H}+1\right)}, \mathbf{N}_{n}=n\left[\begin{array}{ll}
0 & 1 \\
1 & 0
\end{array}\right], n=1,2, \ldots, N_{H}
$$

The $\mathbf{E}$ and $\mathbf{E}^{-1}$ transformation matrices used in eqs. (6) and (20) are:

$$
\mathbf{E}=\frac{2}{2 N_{H+1}}\left[\begin{array}{cccc}
1 / 2 & 1 / 2 & \ldots & 1 / 2 \\
\cos t_{0} & \cos t_{1} & \ldots & \cos t_{2 N_{H}} \\
\sin t_{0} & \sin t_{1} & \ldots & \sin t_{2 N_{H}} \\
\cos 2 t_{0} & \cos 2 t_{1} & \ldots & \cos 2 t_{2 N_{H}} \\
\sin 2 t_{0} & \sin 2 t_{1} & \ldots & \sin 2 t_{2 N_{H}} \\
\vdots & \vdots & & \vdots \\
\cos N_{H} t_{0} & \cos N_{H} t_{1} & \ldots & \cos N_{H} t_{2 N_{H}} \\
\sin N_{H} t_{0} & \sin N_{H} t_{1} & \ldots & \sin N_{H} t_{2 N_{H}}
\end{array}\right]
$$

and

$$
\mathbf{E}^{-1}=\left[\begin{array}{cccccc}
1 & \cos t_{0} & \sin t_{0} & \ldots & \cos N_{H} t_{0} & \sin N_{H} t_{0} \\
1 & \cos t_{1} & \sin t_{1} & \ldots & \cos N_{H} t_{1} & \sin N_{H} t_{1} \\
\vdots & \vdots & \vdots & & \vdots & \vdots \\
1 & \cos t_{2 N_{H}} & \sin t_{2 N_{H}} & \ldots & \cos N_{H} t_{2 N_{H}} & \sin N_{H} t_{2 N_{H}}
\end{array}\right]
$$

$\hat{\mathbf{Q}}_{w_{i}}, i=(1,2,3,4)$ denote the Fourier coefficients of the aerodynamic force generalising variables from system (19) where:

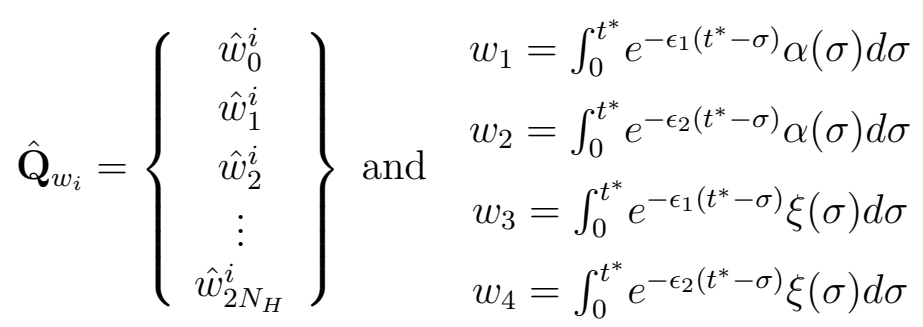


where $\epsilon_{1}=0.0455$ and $\epsilon_{2}=0.3$, also $\psi_{1}=0.165$ and $\psi_{2}=0.335$. The matrices $\mathbf{A}_{1}, \mathbf{B}_{1}, \mathbf{A}_{2}$ and $\mathbf{B}_{2}$ from system (20) are given as:

$$
\begin{aligned}
& \mathbf{A}_{1}=c_{1} \omega^{2} \mathbf{A}^{2}+c_{3} \omega \mathbf{A}+c_{6} \mathbf{I}+c_{8}\left(\omega \mathbf{A}+\epsilon_{1} \mathbf{I}\right)^{-1}+c_{9}\left(\omega \mathbf{A}+\epsilon_{2} \mathbf{I}\right)^{-1} \text { (A.1) } \\
& \mathbf{B}_{1}=c_{0} \omega^{2} \mathbf{A}^{2}+c_{2} \omega \mathbf{A}+c_{4} \mathbf{I}+c_{10}\left(\omega \mathbf{A}+\epsilon_{1} \mathbf{I}\right)^{-1}+c_{11}\left(\omega \mathbf{A}+\epsilon_{2} \mathbf{I}\right)^{-1}(\mathrm{~A} .2) \\
& \mathbf{A}_{2}=d_{1} \omega^{2} \mathbf{A}^{2}+d_{2} \omega \mathbf{A}+d_{3} \mathbf{I}+d_{7}\left(\omega \mathbf{A}+\epsilon_{1} \mathbf{I}\right)^{-1}+d_{8}\left(\omega \mathbf{A}+\epsilon_{2} \mathbf{I}\right)^{-1} \text { (A.3) } \\
& \mathbf{B}_{2}=d_{0} \omega^{2} \mathbf{A}^{2}+d_{5} \omega \mathbf{A}+d_{6} \mathbf{I}+d_{9}\left(\omega \mathbf{A}+\epsilon_{1} \mathbf{I}\right)^{-1}+d_{10}\left(\omega \mathbf{A}+\epsilon_{2} \mathbf{I}\right)^{-1}(\mathrm{~A} .4)
\end{aligned}
$$

\section{Appendix B. Modal Models}

The aeroelastic mass, damping and stiffness matrices are given as:

$$
\begin{aligned}
{\left[\tilde{\mathbf{M}}_{\phi}\right] } & =\left[\mathbf{M}_{\phi}-\frac{\rho V^{2}}{2}\left(\frac{b}{V}\right)^{2} \mathbf{A}_{2}\right] \\
{\left[\tilde{\mathbf{C}}_{\phi}\right] } & =\left[\mathbf{C}_{\phi}-\frac{\rho V^{2}}{2} \frac{b}{V} \mathbf{A}_{1}\right] \\
{\left[\tilde{\mathbf{K}}_{\phi}\right] } & =\left[\mathbf{K}_{\phi}-\frac{\rho V^{2}}{2} \mathbf{A}_{0}\right]
\end{aligned}
$$

The nonlinear restoring force in the spatial domain for the supercritical and subcritical cases respectively are given as:

$$
\mathbf{K}_{n l}(\mathbf{q})=\left\{\begin{array}{c}
0 \\
\vdots \\
0 \\
-\beta_{z}\left(\left[\mathbf{\Phi}\left(q_{1},:\right)\right]\{\mathbf{q}\}-\left[\mathbf{\Phi}\left(q_{2},:\right)\right]\{\mathbf{q}\}\right)^{3} \\
-\beta_{r y}\left(\left[\mathbf{\Phi}\left(q_{3},:\right)\right]\{\mathbf{q}\}-\left[\mathbf{\Phi}\left(q_{4},:\right)\right]\{\mathbf{q}\}\right)^{3} \\
0 \\
\vdots \\
0 \\
\beta_{z}\left(\left[\mathbf{\Phi}\left(q_{1},:\right)\right]\{\mathbf{q}\}-\left[\mathbf{\Phi}\left(q_{2},:\right)\right]\{\mathbf{q}\}\right)^{3} \\
\beta_{r y}\left(\left[\mathbf{\Phi}\left(q_{3},:\right)\right]\{\mathbf{q}\}-\left[\mathbf{\Phi}\left(q_{4},:\right)\right]\{\mathbf{q}\}\right)^{3} \\
0 \\
\vdots \\
0
\end{array}\right\}
$$


and

$$
\mathbf{K}_{n l}(\mathbf{q})=\left\{\begin{array}{c}
0 \\
\vdots \\
0 \\
-\gamma_{z}\left(\left[\mathbf{\Phi}\left(q_{1},:\right)\right]\{\mathbf{q}\}-\left[\mathbf{\Phi}\left(q_{2},:\right)\right]\{\mathbf{q}\}\right)^{5}+\beta_{z}\left(\left[\mathbf{\Phi}\left(q_{1},:\right)\right]\{\mathbf{q}\}-\left[\mathbf{\Phi}\left(q_{2},:\right)\right]\{\mathbf{q}\}\right)^{3} \\
-\gamma_{r y}\left(\left[\mathbf{\Phi}\left(q_{3},:\right)\right]\{\mathbf{q}\}-\left[\mathbf{\Phi}\left(q_{4},:\right)\right]\{\mathbf{q}\}\right)^{5}+\beta_{r y}\left(\left[\mathbf{\Phi}\left(q_{3},:\right)\right]\{\mathbf{q}\}-\left[\mathbf{\Phi}\left(q_{4},:\right)\right]\{\mathbf{q}\}\right)^{3} \\
0 \\
\vdots \\
0 \\
\gamma_{z}\left(\left[\mathbf{\Phi}\left(q_{1},:\right)\right]\{\mathbf{q}\}-\left[\mathbf{\Phi}\left(q_{2},:\right)\right]\{\mathbf{q}\}\right)^{5}-\beta_{z}\left(\left[\mathbf{\Phi}\left(q_{1},:\right)\right]\{\mathbf{q}\}-\left[\mathbf{\Phi}\left(q_{2},:\right)\right]\{\mathbf{q}\}\right)^{3} \\
\gamma_{r y}\left(\left[\mathbf{\Phi}\left(q_{3},:\right)\right]\{\mathbf{q}\}-\left[\mathbf{\Phi}\left(q_{4},:\right)\right]\{\mathbf{q}\}\right)^{5}-\beta_{r y}\left(\left[\mathbf{\Phi}\left(q_{3},:\right)\right]\{\mathbf{q}\}-\left[\mathbf{\Phi}\left(q_{4},:\right)\right]\{\mathbf{q}\}\right)^{3} \\
0 \\
\vdots \\
0
\end{array}\right\}
$$

The transformation matrices used in eq. (24) are given as:

$$
\begin{aligned}
& \mathbf{E}_{d e f}^{-1}=\left[\begin{array}{cccc}
{\left[\mathbf{e}_{d e f}\right]_{1}} & \mathbf{0} & \ldots & \mathbf{0} \\
\mathbf{0} & {\left[\mathbf{e}_{d e f}\right]_{2}} & \ddots & \vdots \\
\vdots & \ddots & \ddots & \mathbf{0} \\
\mathbf{0} & \ldots & \mathbf{0} & {\left[\mathbf{e}_{d e f}\right]_{n_{m}}}
\end{array}\right],\left[\mathbf{e}_{d e f}\right]=\left[\begin{array}{lll}
1 & \cos \omega t \ldots \sin N_{H} \omega t
\end{array}\right] \\
& \mathbf{E}_{v e l}^{-1}=\left[\begin{array}{cccc}
{\left[\mathbf{e}_{v e l}\right]_{1}} & \mathbf{0} & \ldots & \mathbf{0} \\
\mathbf{0} & {\left[\mathbf{e}_{v e l}\right]_{2}} & \ddots & \vdots \\
\vdots & \ddots & \ddots & \mathbf{0} \\
\mathbf{0} & \ldots & \mathbf{0} & {\left[\mathbf{e}_{v e l}\right]_{n_{m}}}
\end{array}\right],\left[\mathbf{e}_{v e l}\right]=\left[0-\omega \sin \omega t \ldots \omega N_{H} \cos N_{H} \omega t\right] \\
& \mathbf{E}_{a c c}^{-1}=\left[\begin{array}{cccc}
{\left[\mathbf{e}_{a c c}\right]_{1}} & \mathbf{0} & \ldots & \mathbf{0} \\
\mathbf{0} & {\left[\mathbf{e}_{a c c}\right]_{2}} & \ddots & \vdots \\
\vdots & \ddots & \ddots & \mathbf{0} \\
\mathbf{0} & \ldots & \mathbf{0} & {\left[\mathbf{e}_{a c c}\right]_{n_{m}}}
\end{array}\right],\left[\mathbf{e}_{v e l}\right]=\left[\begin{array}{ll}
\left.0-\omega^{2} \cos \omega t \ldots-\left(\omega N_{H}\right)^{2} \sin N_{H} \omega t\right] \\
\end{array}\right.
\end{aligned}
$$


The convolution integral of the inverse Laplace transform of the generalised aerodynamic matrix yields:

$$
\dot{\mathbf{q}}_{a_{i}}=\int_{0}^{t} \dot{\mathbf{q}} e^{-\frac{V}{b} \eta_{i}\left(t-t^{*}\right)} d t^{*}, \quad i=1, \ldots, n_{l}
$$

Evaluating the integral gives

$$
\dot{\mathbf{q}}_{a_{i}}=\dot{\mathbf{q}} \frac{b}{V \eta_{i}}\left(1-e^{-\frac{V}{b} \eta_{i} t}\right)
$$

Here we are interested in the periodic solution only so the exponential term is damped out which yields:

$$
\dot{\mathbf{q}}_{a_{i}}=\dot{\mathbf{q}} \frac{b}{V \eta_{i}}, \quad i=1, \ldots, n_{l}
$$

The discrete Fourier transforms used in eq. (24) for the $j$ th mode can be given as:

$$
\begin{gathered}
\hat{q}_{0}^{a_{i}, j}=\frac{1}{2 \pi} \int_{0}^{2 \pi} \frac{b}{V \eta_{i}}\left(\sum_{n=1}^{N_{H}}\left(-\omega n \hat{q}_{2 n-1}^{j} \sin \omega n t+\omega n \hat{q}_{2 n}^{j} \cos \omega n t\right)\right) d t \\
\hat{q}_{2 m-1}^{a_{i}, j}=\frac{1}{\pi} \int_{0}^{2 \pi} \frac{b}{V \eta_{i}}\left(\sum_{n=1}^{N_{H}}\left(-\omega n \hat{q}_{2 n-1}^{j} \sin \omega n t+\omega n \hat{q}_{2 n}^{j} \cos \omega n t\right)\right) \cos m t d t \\
\hat{q}_{2 m}^{a_{i}, j}=\frac{1}{\pi} \int_{0}^{2 \pi} \frac{b}{V \eta_{i}}\left(\sum_{n=1}^{N_{H}}\left(-\omega n \hat{q}_{2 n-1}^{j} \sin \omega n t+\omega n \hat{q}_{2 n}^{j} \cos \omega n t\right)\right) \sin m t d t \\
\hat{f}_{0}^{j}=\frac{1}{2 \pi} \int_{0}^{2 \pi}[\mathbf{\Phi}]^{\mathrm{T}} \mathbf{K}_{n l}(\mathbf{q}) d t \\
\hat{f}_{2 m}^{j}=\frac{1}{\pi} \int_{0}^{2 \pi}[\mathbf{\Phi} .11]^{\mathrm{T}} \mathbf{K}_{n l}(\mathbf{q}) \cos m t d t
\end{gathered}
$$

for $m=1, \ldots, N_{H},\left\{\hat{\mathbf{f}}_{j}\right\}=\left[\begin{array}{llll}\hat{f}_{0}^{j} & \hat{f}_{1}^{j} & \ldots & \hat{f}_{2 N_{H}+1}^{j}\end{array}\right]^{\mathrm{T}}$ and $\hat{\mathbf{K}}_{n l}=\left[\begin{array}{llll}\hat{\mathbf{f}}_{1} & \hat{\mathbf{f}}_{2} & \ldots & \hat{\mathbf{f}}_{n_{m}}\end{array}\right]^{\mathrm{T}}$. The DFTs are computed numerically due to their complexity using a trapezoidal rule[43]. 


\section{Appendix C. Nomenclature}

\section{Appendix C.1. Roman Symbols}

a polynomial coefficients for polynomial chaos expansions

$b$ semi-chord

C structural damping matrix

$C_{L}$ lift coefficient

$C_{M}$ pitching moment coefficient

E time/frequency domain transformation matrix

F generalised external force matrix

J Newton-Raphson system Jacobian

$k$ linear stiffness coefficient

K structural linear stiffness matrix

$m$ structural mass

$M$ number of independent continuous uncertain parameters

M structural mass matrix

$n_{l}$ Goland wing, number of lag variables in rational approximation

$N_{H}$ number of harmonics

$\mathbf{q}$ vector of modal deflections

$\mathbf{q}_{a_{i}}$ decomposed generalised aerodynamic vectors, $i=1, \ldots, n_{l}$

Q solution vector

$r_{\alpha}$ radius of gyration

$\mathbf{R}^{\mathbf{n}}$ Newton-Raphson system residual vector at iteration $n$

$\mathbf{S}^{\mathbf{n}}$ Newton-Raphson solution vector at iteration $n$ 
$t$ time

$V$ freestream velocity

$w_{i}$ Wagner variables, $i=1,2,3,4$

$x$ solution

$x_{\alpha}$ distance between mass centre and elastic axis

$\hat{x}_{i}$ Fourier coefficient of displacement, $i=1, \ldots, N_{H}$

Appendix C.2. Greek Symbols

$\alpha$ aerofoil pitch displacement, angle-of-attack

$\beta$ cubic stiffness coefficient

$\gamma$ pentic stiffness coefficient

$\Gamma$ polynomial chaos basis functions

$\epsilon_{i}$ constants in Wagner's function, $i=1,2$

$\varphi$ polynomial chaos random variable

$\zeta$ damping ratio

$\eta$ Goland wing, term from rational approximation of generalised aerodynamic forces, $i=1, \ldots, n_{l}$

$\lambda$ Newton-Raphson relaxation parameter

$\mu$ aerofoil air mass ratio

$\xi$ aerofoil non-dimensionalised plunge displacement

$\rho$ air density

$\Phi$ truncated matrix of eigenvectors

$\theta$ uncertain parameters

$\omega$ fundamental solution frequency

$\bar{\omega}$ aerofoil, frequency ratio, $\bar{\omega}=\omega_{\xi} / \omega_{\alpha}$ 
Appendix C.3. Subscripts, Superscripts and Oversets

()$_{a_{i}}$ decomposed generalised aerodynamic vectors

()$_{L}$ coefficient of lift

()$_{M}$ coefficient of pitching moment

()$_{n l}$ nonlinear force

()$_{r y}$ rotation about $y$ axis $d o f$

()$_{w_{i}}$ Wagner function representative aerodynamic variables

()$_{x}$ Duffing oscillator displacement

()$_{z}$ translation about $z$ axis dof

()$_{\alpha}$ aerofoil pitch $d o f$

()$_{\xi}$ aerofoil plunge $d o f$

()$_{\phi}$ quantity in modal domain

()$_{0}$ initial condition (when specified)

()$^{*}$ non-dimensionalised quantity

() Fourier coefficient

() equally spaced time domain solution

[1] T. Noll, J. Brown, M. Perez-Davis, S. Ishmael, G. Tiffany, M. Gaier, Investigation of the Helios Prototype Aircraft Mishap, Tech. rep., NASA (2004).

[2] C. Pettit, Uncertainty Quantification in Aeroelasticity: Recent Results and Research Challenges, Journal of Aircraft 41 (5) (2004) 1217-1229.

[3] S. Marques, K. Badcock, H. Khodaparast, J. Mottershead, Transonic Aeroelastic Stability Predictions Under the Influence of Structural Variability, Journal of Aircraft 47 (4) (2010) 1229-1239. 
[4] S. Marques, K. Badcock, H. Khodaparast, J. Mottershead, How Structural Model Variability Influences Transonic Aeroelastic Stability, Journal of Aircraft 49 (5), doi:10.2514/1.C031103.

[5] R. Bunton, C. Denegri, Limit Cycle Oscillation Characteristics of Fighter Aircraft, Journal of Aircraft 37 (5) (2000) 916-918.

[6] J. Thomas, E. Dowell, K. Hall, C. Denegri, Modeling Limit Cycle Oscillation Behavior of the F-16 Fighter Using a Harmonic Balance Approach, no. AIAA-2004-1696, 2004, presented at the AIAA/ASME/ASCE/AHS/ASC Structures, Structural Dynamics, and Materials Conference.

[7] B. Stanford, P. Beran, Direct flutter and limit cycle computations of highly flexible wings for efficient analysis and optimization, Journal of Fluids and Structures 36 (0) (2013) 111-123, doi:10.1016/j.jfluidstructs.2012.08.008.

[8] P. Beran, D. Lucia, C. Pettit, Reduced-order modelling of limit-cycle oscillation for aeroelastic systems, Journal of Fluids and Structures 19 (5) (2004) 575-590, doi:10.1016/j.jfluidstructs.2004.04.002.

[9] T. Lieu, C. Farhat, M. Lesionne, Reduced-Order Fluid/Structure Modeling of a Complete Aircraft Configuration, Computer Methods in Applied Mechanics and Engineering 195 (2006) 5730-5742.

[10] B. Stanford, P. Beran, Computational strategies for reliability-based structural optimization of aeroelastic limit cycle oscillations, Structural and Multidisciplinary Optimization 45 (2012) 83-99.

[11] S. Munteanu, J. Rajadas, C. Nam, A. Chattopadhyay, Reduced-ordermodel approach for aeroelastic analysis involving aerodynamic and structural nonlinearities, AIAA Journal 43 (3) (2005) 560-571.

[12] W. Yao, M.-S. Liou, Reduced-Order Modeling for Flutter/LCO Using Recurrent Artificial Neural NetworkPresented at the 12th AIAA Aviation, Technology, Integration and Operations (ATIO) Conference, Indianapolis, Indiana.

[13] G. Vio, G. Dimitriadis, J. Cooper, K. Badcock, M. Woodgate, A. Rampurawala, Aeroelastic system identification using transonic CFD data 
for a wing/store configuration, Aerospace Science and Technology 11 (2007) 146-154.

[14] T. Kim, System identification for coupled fluid-structures:Aerodynamics is aeroelasticity minus structure, AIAA Journal 49 (3) (2011) 503-512.

[15] K. Badcock, H. Khodaparast, S. Timme, J. Mottershead, Calculating the Influence of Structural Uncertainty on Aeroelastic Limit Cycle ResponsePresented at the 52nd AIAA/ASME/ASCE/AHS/ASC Structures, Structural Dynamics, and Materials Conference.

[16] P. Beran, C. Pettit, A Direct Method for Quantifying Limit-Cycle Oscillation Response Characteristics in the Presence of Uncertainties (AIAA2004-1695), presented at the 45th AIAA/ASME/ASCE/AHS/ASC Structures, Structural Dynamics, and Materials Conference, Palm Springs, California.

[17] B. Stanford, P. Beran, Limit Cycle Oscillations of a StructurallyOptimized Cantilevered Wing (AIAA-2010-9124), presented at the 13th AIAA/ISSMO Multidisciplinary Analysis Optimization Conference, Fort Worth, Texas.

[18] L. Liu, E. Dowell, J. Thomas, A high dimensional harmonic balance approach for an aeroelastic airfoil with cubic restoring forces, Journal of Fluids and Structures 23 (3) (2007) 351-363, doi:10.1016/j.jfluidstructs.2006.09.005.

[19] G. Dimitriadis, G. Vio, J. Cooper, Application of Higher-Order Harmonic Balance to Non-Linear Aeroelastic Systems (AIAA-20062023), presented at the 47th AIAA/ASME/ASCE/AHS/ASC Structures, Structural Dynamics, and Materials Conference, Newport, Rhode Island.

[20] A. Gopinath, P. Beran, A. Jameson, Comparative Analysis of Computational Methods for Limit-Cycle Oscillations, Presented at 47th AIAA/ASME/ASCE/AHS/ASC Structures, Structural Dynamics, and Materials Conference.

[21] G. Dimitriadis, Continuation of Higher-Order Harmonic Balance Solutions for Nonlinear Aeroelastic Systems, Journal of Aircraft 45 (2) (2008) 523-537, doi:10.2514/1.30472. 
[22] K. Hall, J. Thomas, W. Clark, Computation of Unsteady Nonlinear Flows in Cascades Using a Harmonic Balance Technique, AIAA Journal 40 (5) (2002) 879-886.

[23] M. A. Woodgate, G. N. Barakos, Implicit Computational Fluid Dynamics Methods for Fast Analysis of Rotor Flows, AIAA Journal, Vol. 50, No. 6 (2012), pp. 1217-1244.

[24] P. Beran, C. Pettit, D. Millman, Uncertainty Quantification of LimitCycle Oscillations, Journal of Computational Physics 217 (1) (2006) 217-247.

[25] J. A. Witteveen, A. Loeven, S. Sarkar, H. Bijl, Probabilistic collocation for period-1 limit cycle oscillations, Journal of Sound and Vibration 311 (1-2) (2008) 421-439, doi:10.1016/j.jsv.2007.09.017.

[26] J. L. Meitour, D. Lucor, C. Chassaing, Prediction of stochastic limit cycle oscillations using an adaptive Polynomial Chaos method, Journal of Aeroelasticity and Structural Dynamics 2 (1) (2010) 3-22.

[27] L. Liu, J. Thomas, E. Dowell, P. Attar, K. Hall, A comparison of classical and high dimensional harmonic balance approaches for a Duffing oscillator, Journal of Computational Physics 215 (1) (2006) 298-320, doi:10.1016/j.jcp.2005.10.026.

[28] J. P. Thomas, E. H. Dowell, K. C. Hall, Nonlinear Inviscid Aerodynamic Effects on Transonic Divergence, Flutter, and Limit-Cycle Oscillations, AIAA Journal 40 (4) (2002) 638-646, doi: 10.2514/2.1720.

[29] P. S. R. Ghanem, Stochastic Finite Elements: A Spectral Approach, 1st Edition, Springer New York, 1991, doi:10.1007/978-1-4612-3094-6.

[30] O. Le Maître, O. Knio, Non-intrusive Methods, in: Spectral Methods for Uncertainty Quantification, Scientific Computation, Springer Netherlands, 2010, pp. 45-72.

[31] S. Hosder, R. Walters, M. Balch, Point-Collocation Nonintrusive Polynomial Chaos Method for Stochastic Computational Fluid Dynamics, AIAA Journal 48 (12) (2010) 2721-2730, doi: 10.2514/1.39389. 
[32] M. Eldred, Recent Advances in Non-Intusive Polynomial Chaos and Stochastic Collocation Methods for Uncertainty Analysis and Design (AIAA-2009-2274), presented at the AIAA/ASME/ASCE/AHS/ASC Structures, Structural Dynamics, and Materials Conference, Palm Springs, California.

[33] B. Lee, L. Liu, K. Chung, Airfoil motion in subsonic flow with strong cubic nonlinear restoring forces, Journal of Sound and Vibration 218 (35) (2005) 699-717, doi:10.1016/j.jsv.2004.01.034.

[34] B. Lee, L. Gong, Y. Wong, Analysis and Computation of Nonlinear Dynamic Response of a two-Degree-of-Freedom System and its Applications in Aeroelasticity, Journal of Fluids and Structures 11 (1997) 225-246.

[35] B. Lee, L. Jiang, Y. Wong, Flutter of an Airfoil with a Cubic Restoring Force, Journal of Fluids and Structures 13 (jfls.1998.0190) (1998) 75101.

[36] J. L. Meitour, D. Lucor, J. Chassaing, Prediction of stochastic limit cycle oscillations using an adaptive Polynomial Chaos method, Aeroelasticity and Structural Dynamics Journal 2 (1) (2010) 3-22.

[37] D. Millman, P. King, P. Beran, Airfoil Pitch-and-Plunge Bifurcation Behavior with Fourier Chaos Expansions, Journal of Aircraft 42 (2) (2005) 376-384, doi:10.2514/1.5550.

[38] P. Beran, N. Knot, F. Eastep, R. Synder, J. Zweber, Numerical Analysis of Store-Induced Limit Cycle Oscillation, Journal of Aircraft 41 (6) (2004) 1315-1326.

[39] M. Platten, J. Wright, K. Worden, G. Dimitriadis, J. Cooper, Nonlinear identification in modal space using a genetic algorithm approach for model selection, International Journal of Applied Mathematics and Mechanics 3 (1) (2007) 72-89.

[40] M. F. Platten, J. R. Wright, G. Dimitriadis, J. E. Cooper, Identification of multi-degree of freedom non-linear systems using an extended modal space approach, Journal of Mechanical Systems and Signal Processing 23 (2009) 8-29. 
[41] W. Eversman, A. Tewari, Consistent Rational-Function Approximation for Unsteady Aerodynamics, Journal of Aircraft 28 (9) (1991) 545-552.

[42] E. Dowell, R. Clark, D. Cox, H. C. Jr., J. Edwards, K. Hall, D. Peters, R. Scanlan, E. Simiu, F. Sisto, T. Strganac, A Modern Course in Aeroelasticity, Kluwer Academic Publishers, 2004.

[43] K. Atkinson, An Introduction to Numerical Analysis, 2nd Edition, John Wiley and Sons, 1989. 